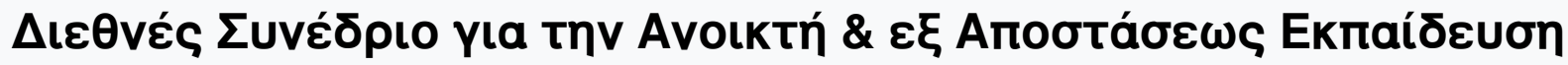

Tón. 6, Ap. 1A (2011)

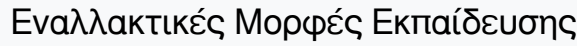

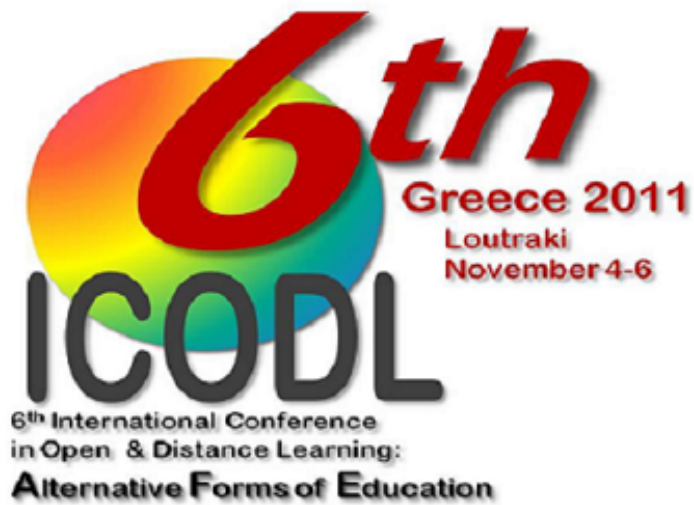

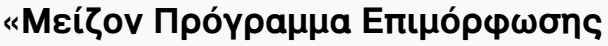

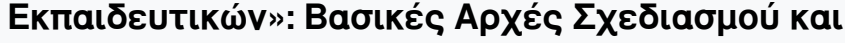

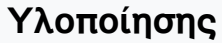

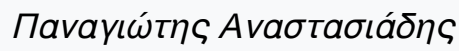

doi: $10.12681 /$ icodl. 686

TOMOEA

PART/MEPOEA 


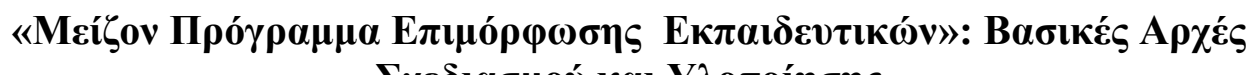

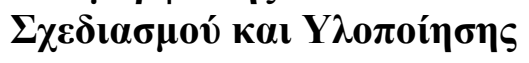

\title{
NATIONAL TEACHER TRAINING PROGRAM OF GREECE: Basic Design and Implementation Principles
}

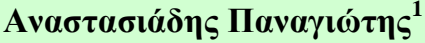

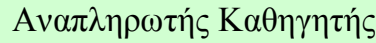

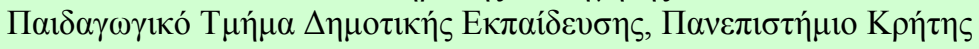 \\ panas@edc.uoc.gr
}

\begin{abstract}
The design of "New School" aims at addressing the contemporary educational needs and challenges of the $21^{\text {st }}$ century. In this context, the "National Program of Teachers' Training" has been designed according to the objectives of the project "Education and Lifelong Learning" of the Ministry of Education, Lifelong Learning and Religion, which aims at upgrading the quality of education. The physical object of the National Teacher Training Program refers at first to the training of 150,000 public and private school teachers. The training is aimed to teachers of all disciplines (primary and secondary education), regardless of the technological tools they have at their disposal and the skills they possess. The educational training program is based on the principles of adult education and intends to ensure maximum flexibility regarding space, time and teachers' special learning rhythms in accordance with the methodology of distance education, while providing for the use of innovative applications from Greece and other countries.
\end{abstract}

\section{Пєрі́ $\lambda \eta \psi \eta$}

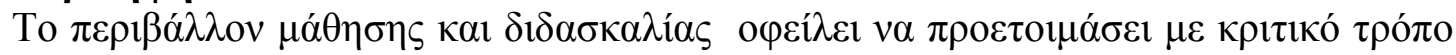

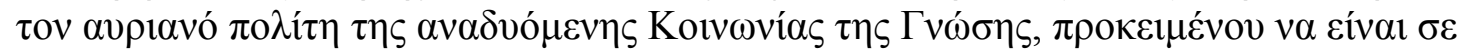

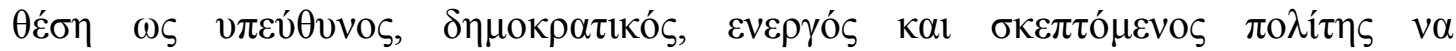

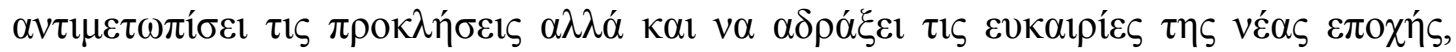

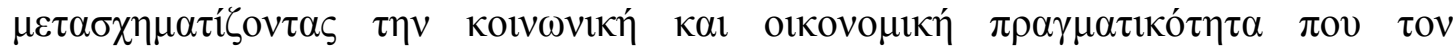

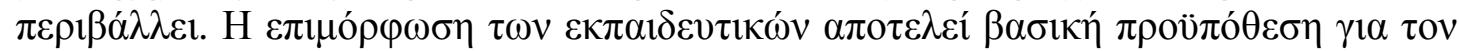

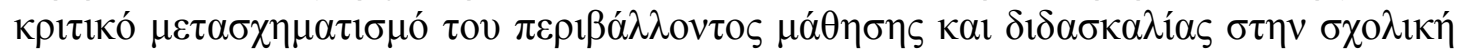

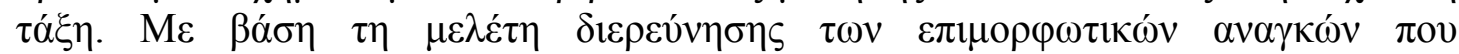

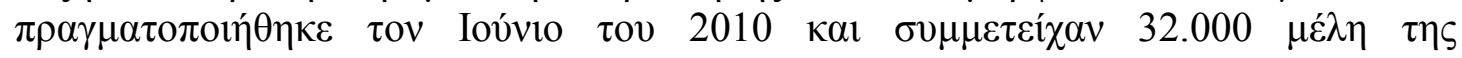

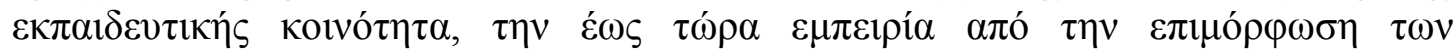

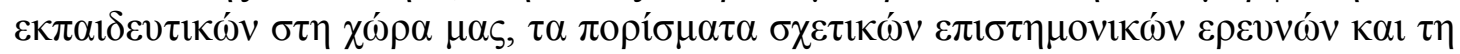

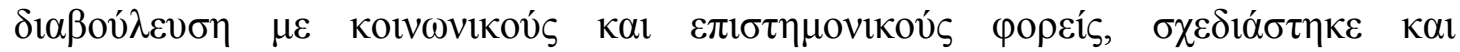

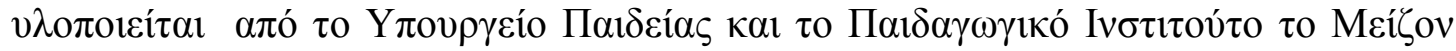

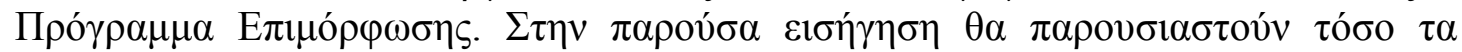

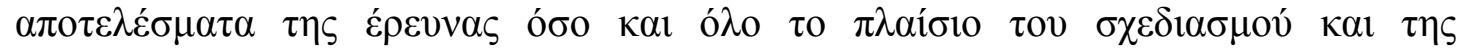

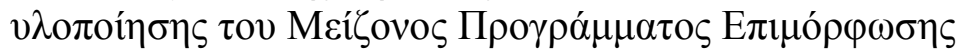

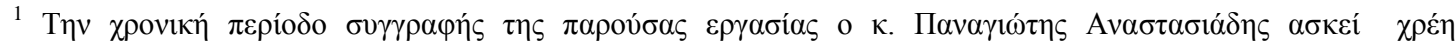

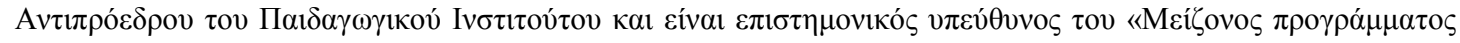

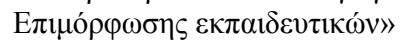




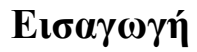

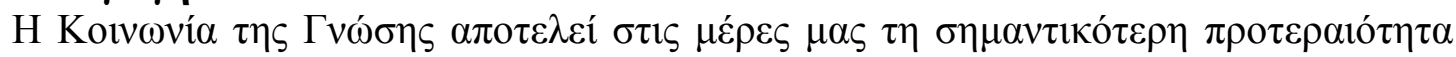

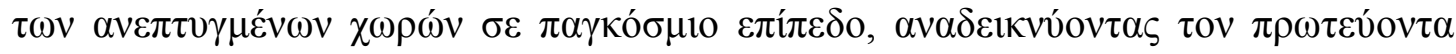

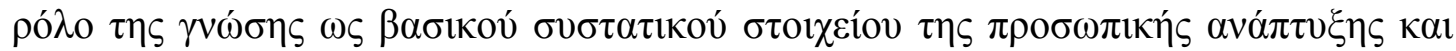

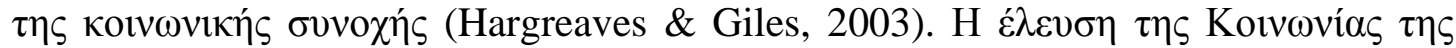

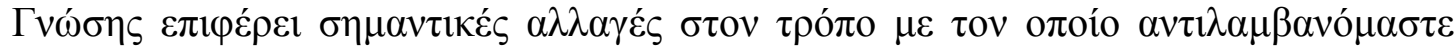

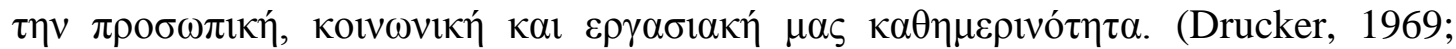

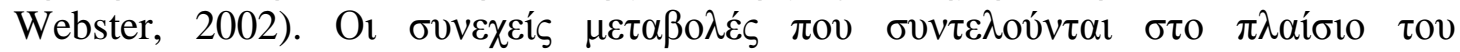

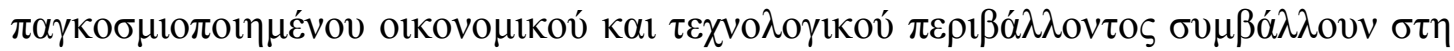

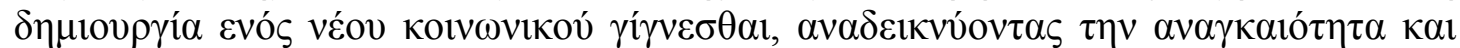

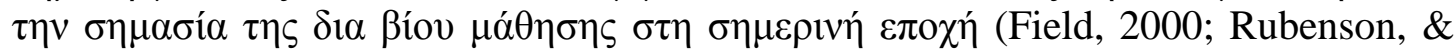

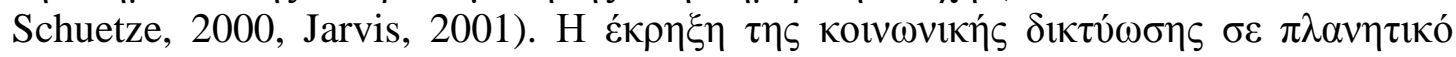

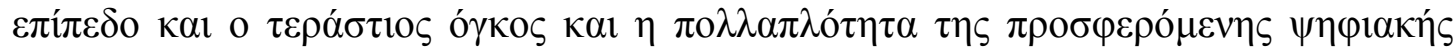

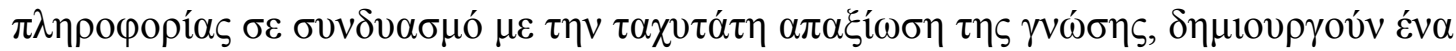

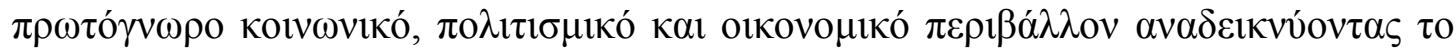

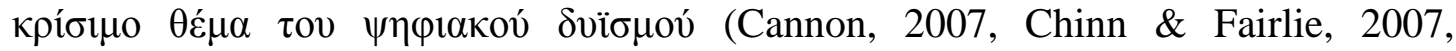

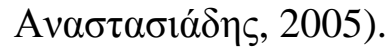

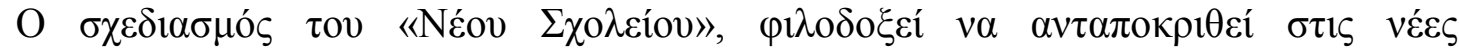

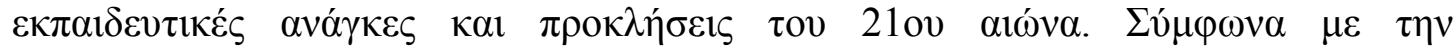

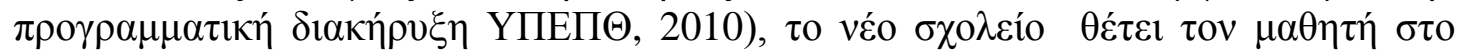

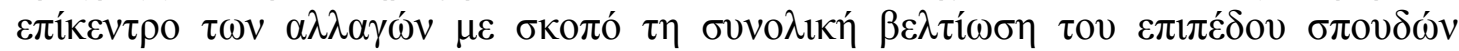

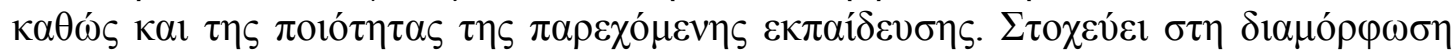

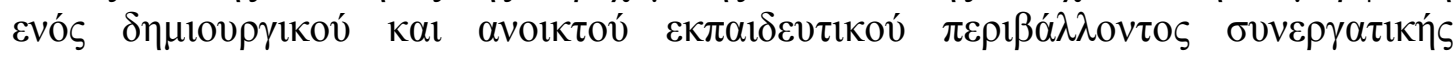

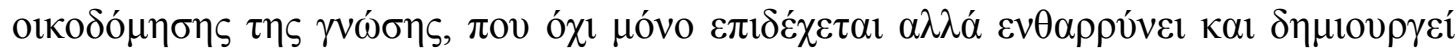

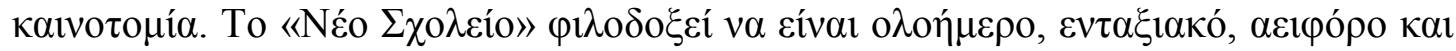

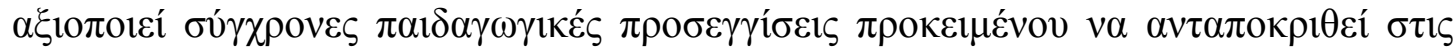

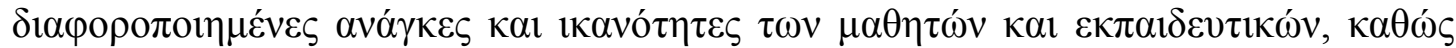

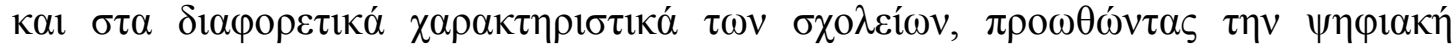
$\varepsilon \pi ı$ เ

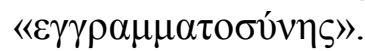

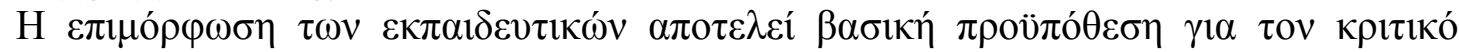

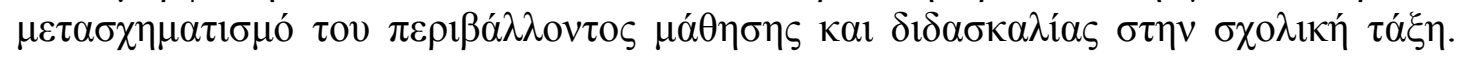

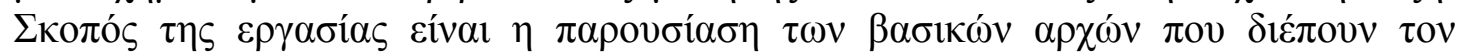

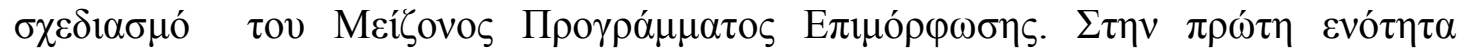

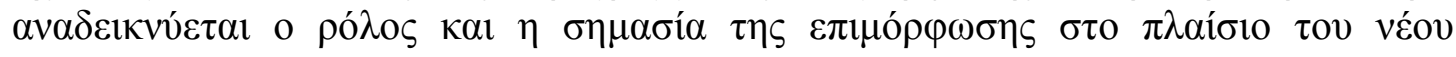

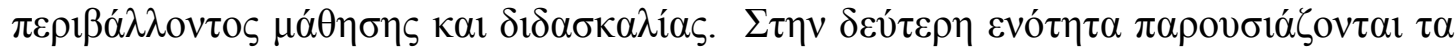

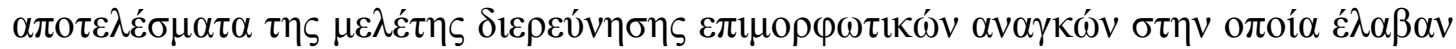

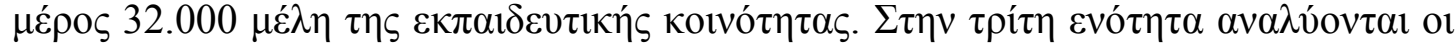

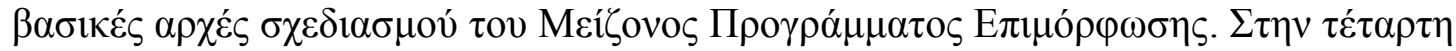

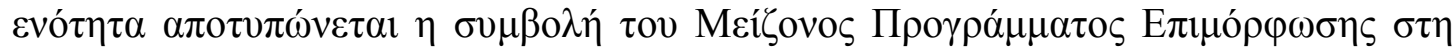

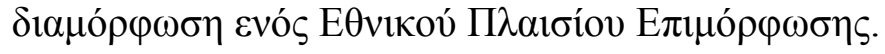

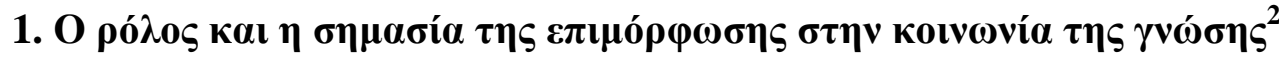

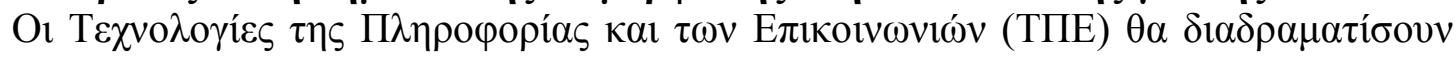

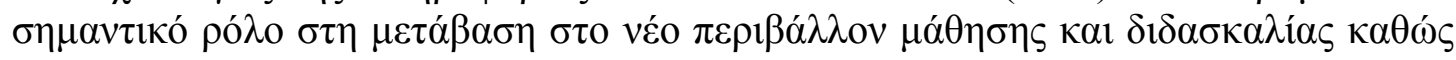

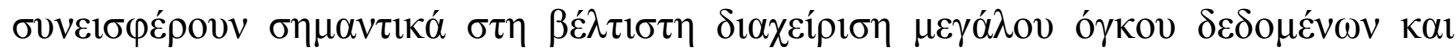

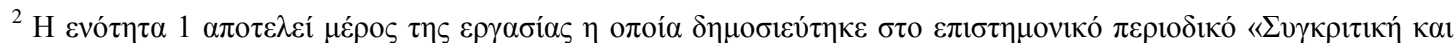

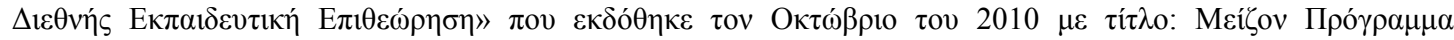

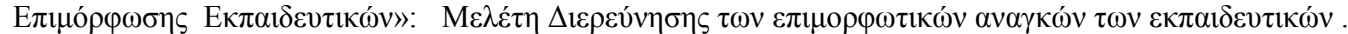




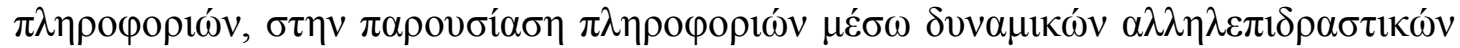

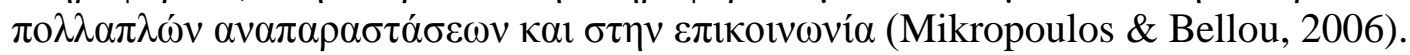

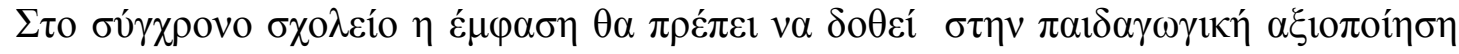

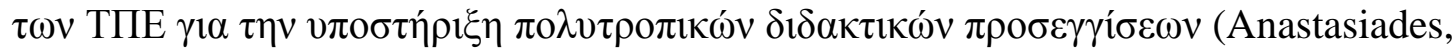

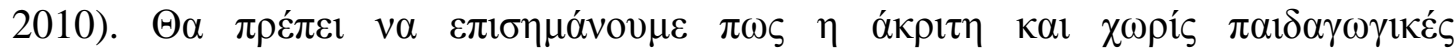

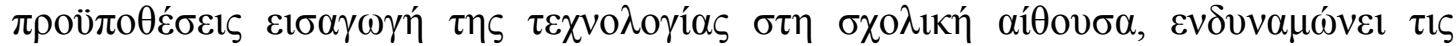

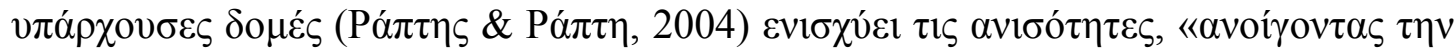

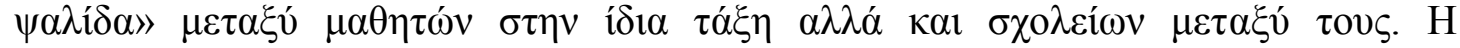

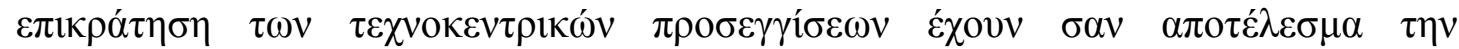

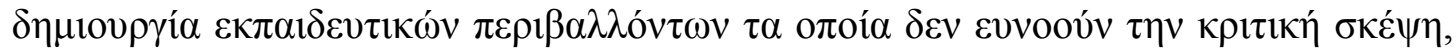

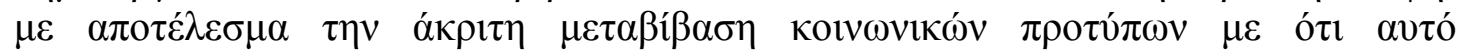

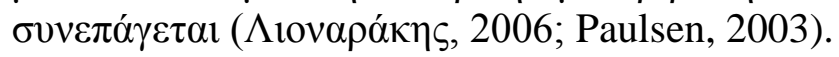

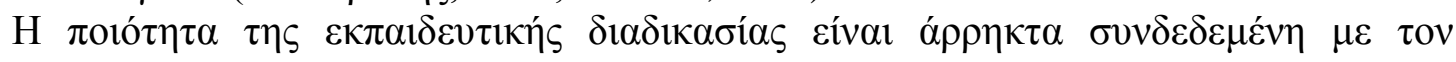

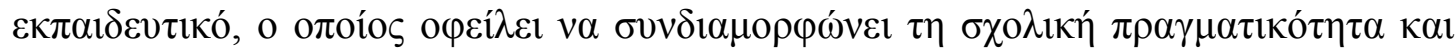

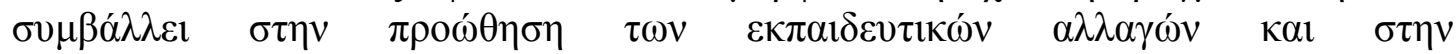

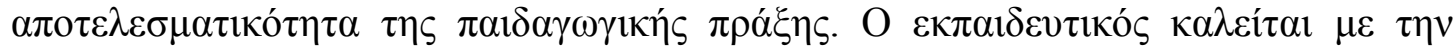

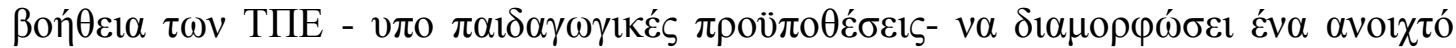

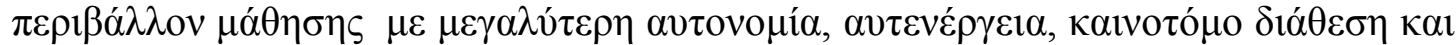

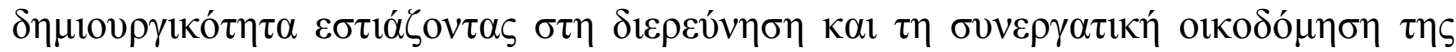

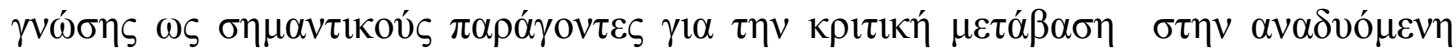

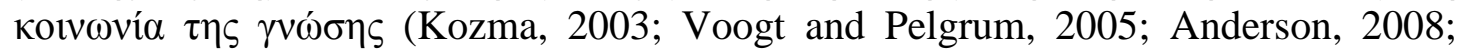
Anastasiades, 2009;).

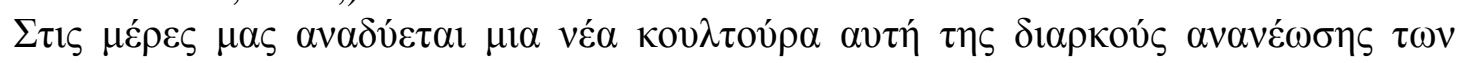

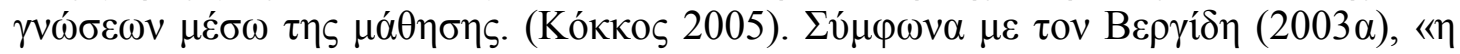

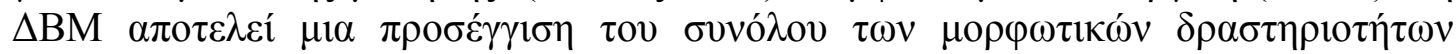

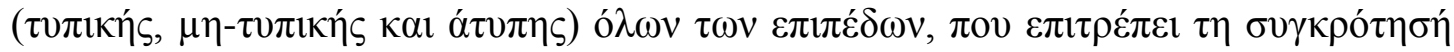

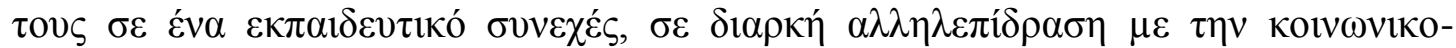

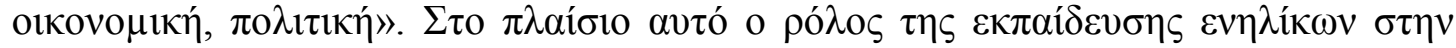

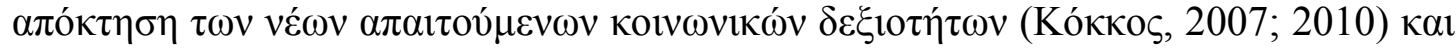

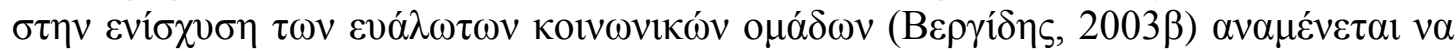

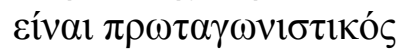

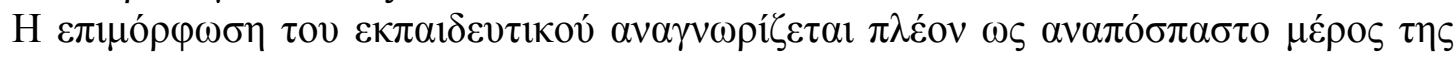

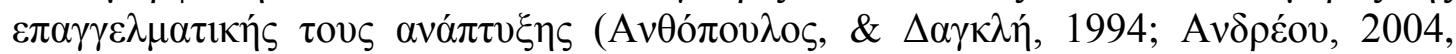

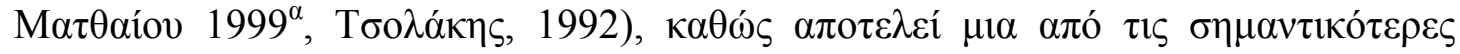

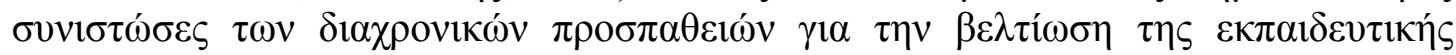

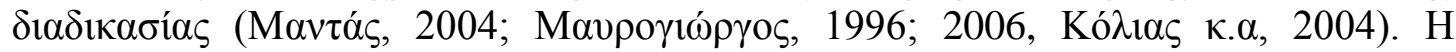

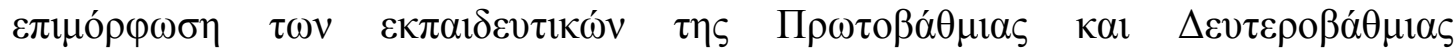

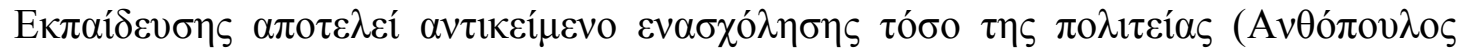

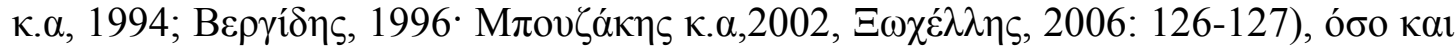

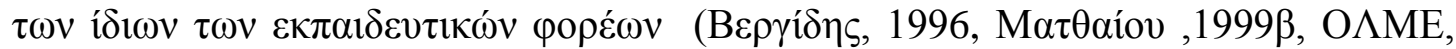

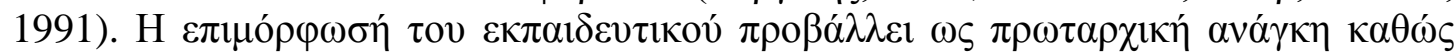

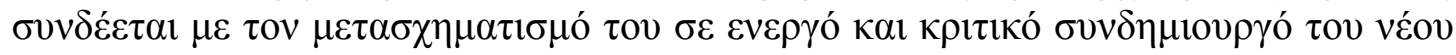

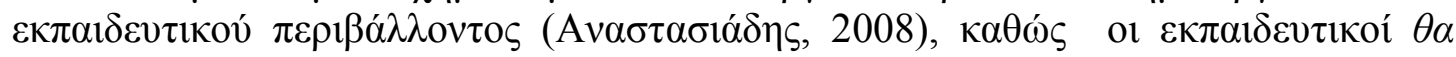

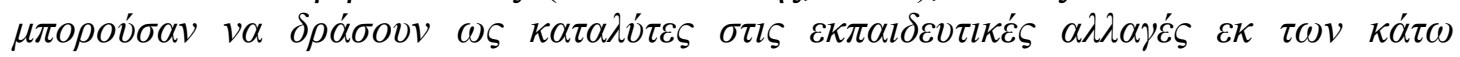

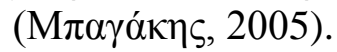

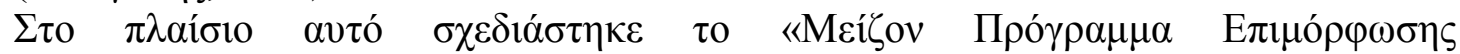

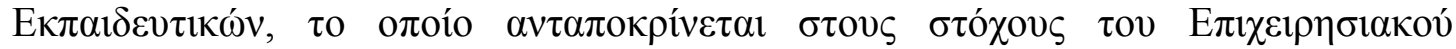

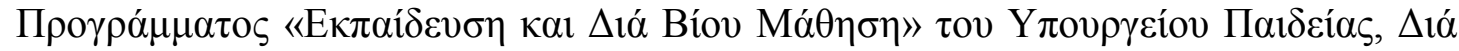

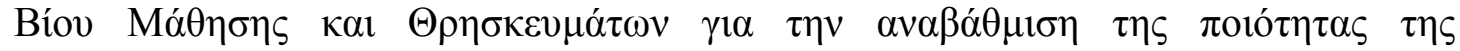

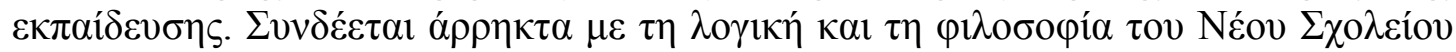




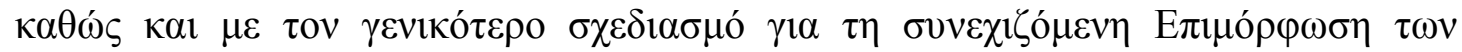

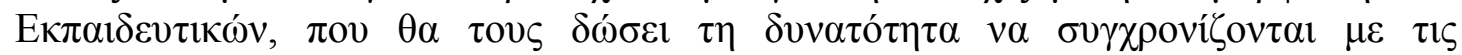

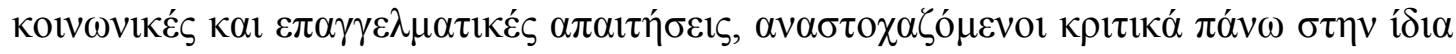

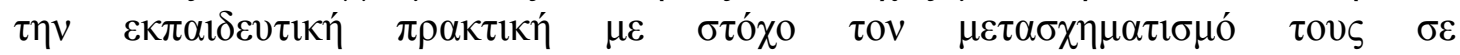

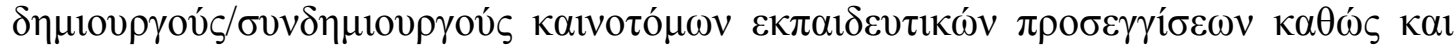

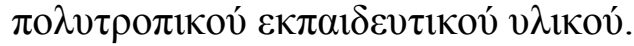

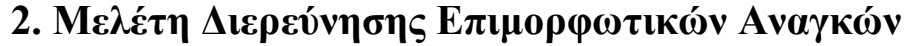

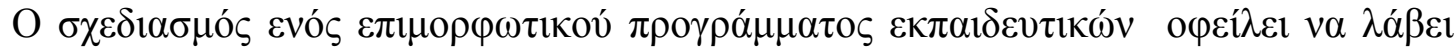

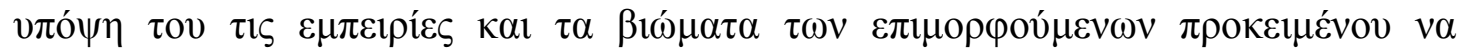

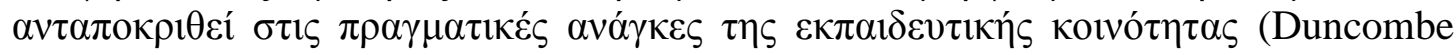

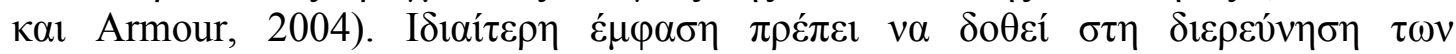

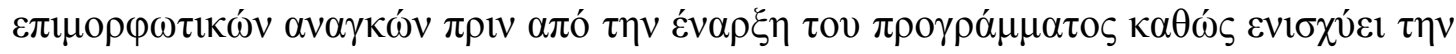

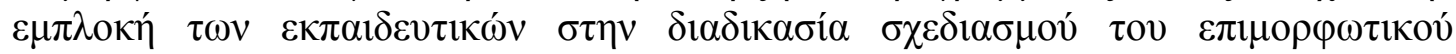

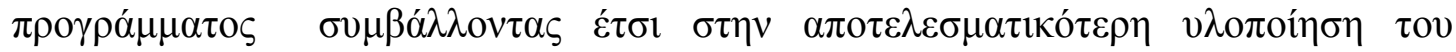
(National Foundation for the Improvement of Education, 1996). $\Sigma v ́ \mu \varphi \omega v \alpha \mu \varepsilon \varepsilon \dot{\varepsilon} \rho \varepsilon v \alpha \alpha$

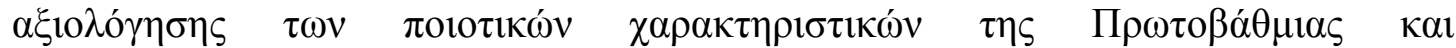

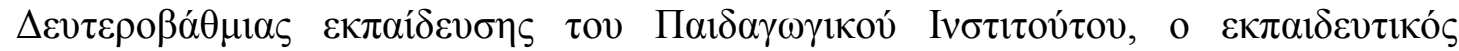

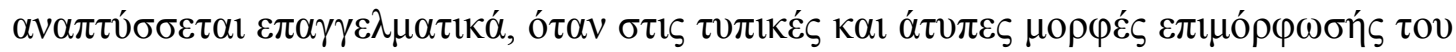

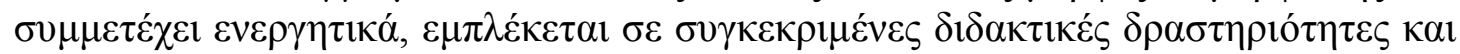

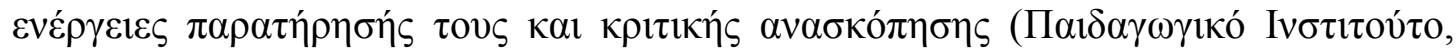

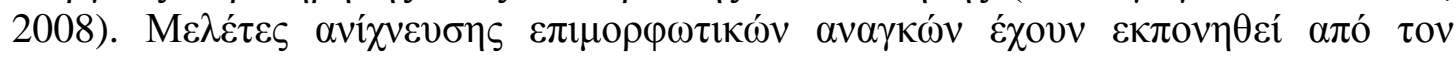

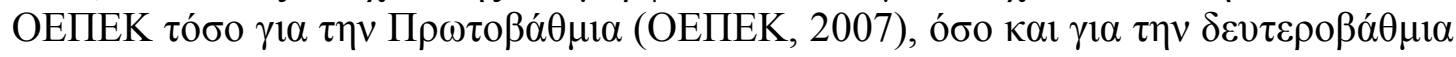
$\varepsilon \kappa \pi \alpha i \delta \varepsilon \varepsilon \sigma \eta$ (OEПЕК, 2008).

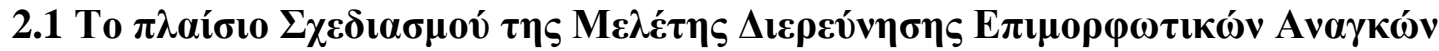

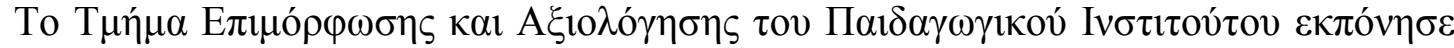

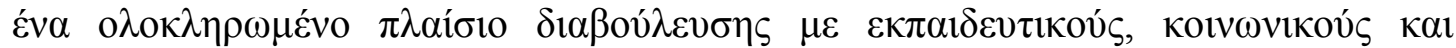

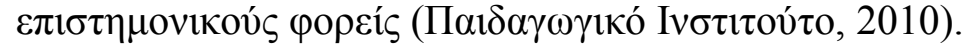

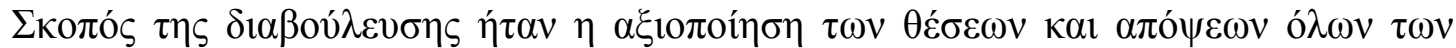

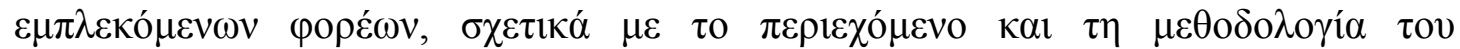

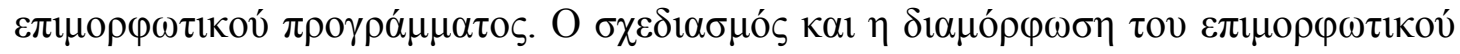

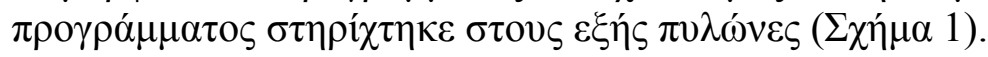

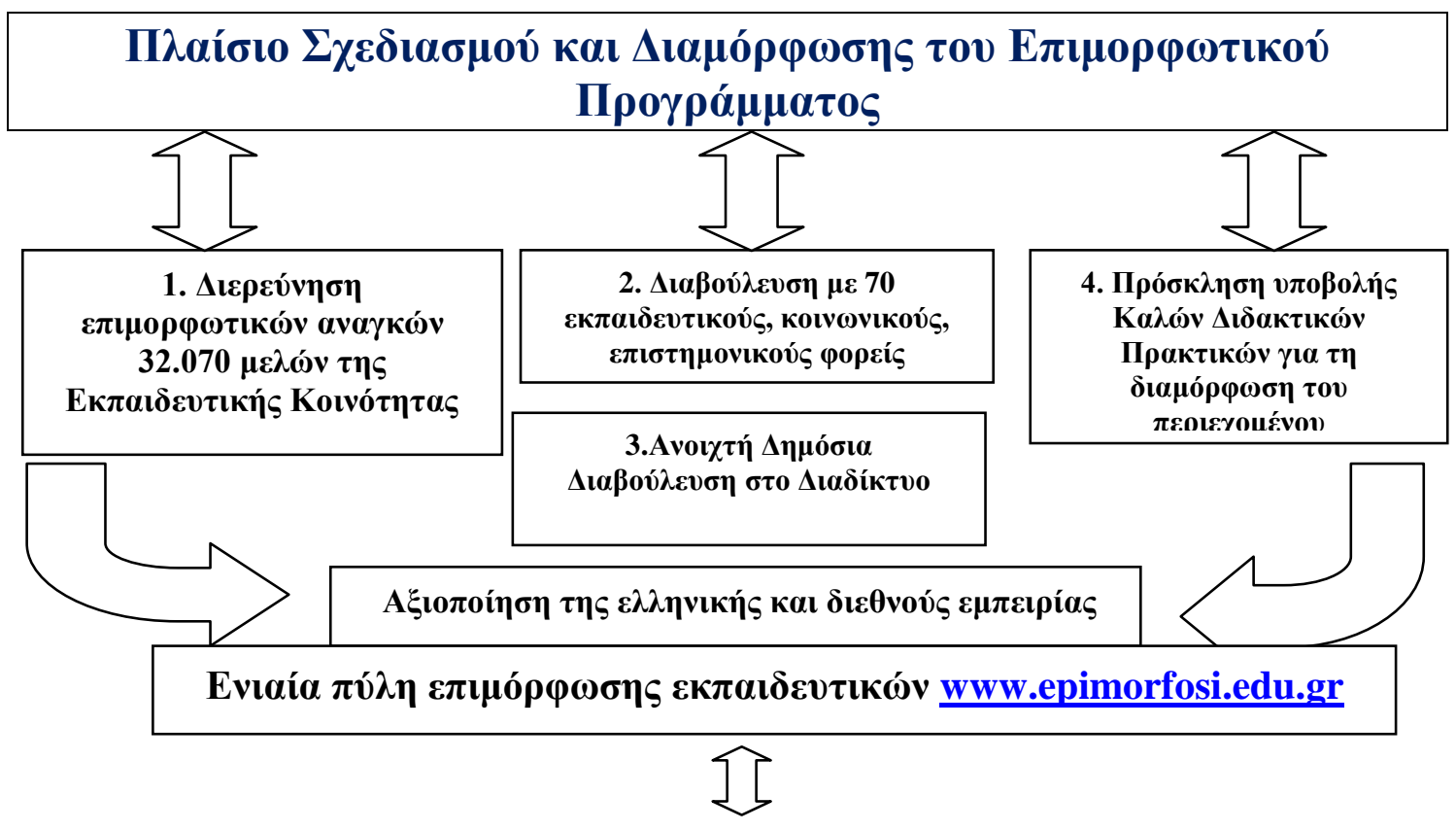




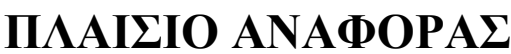

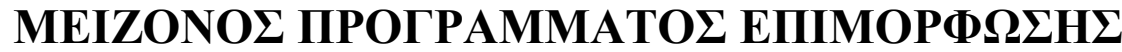

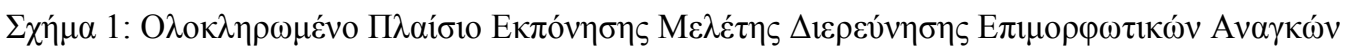

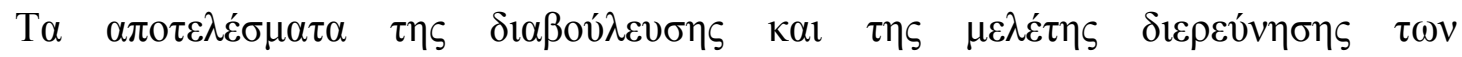

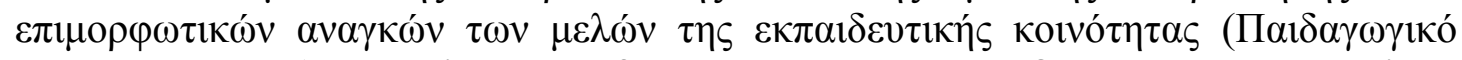

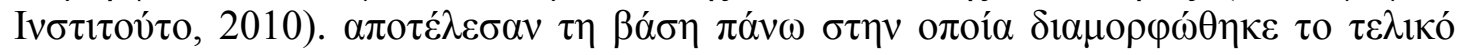

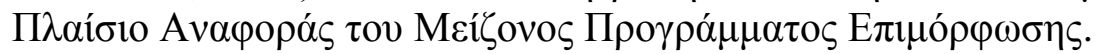

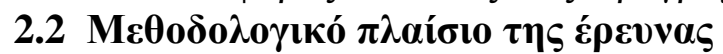

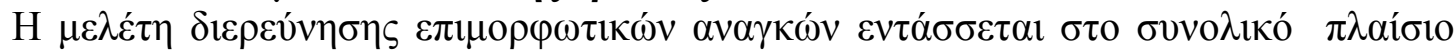

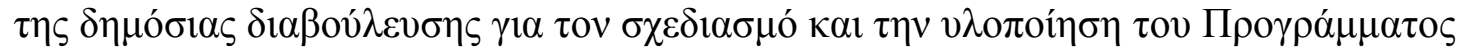

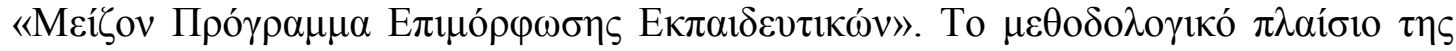

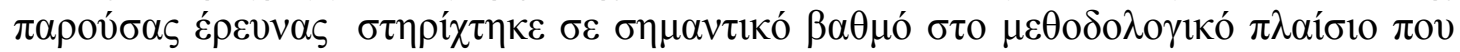

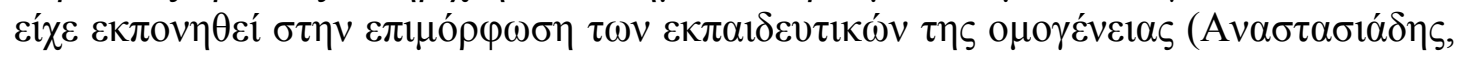

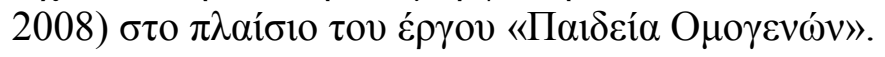

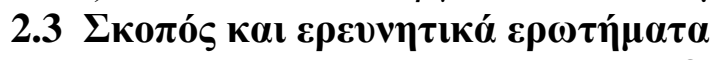

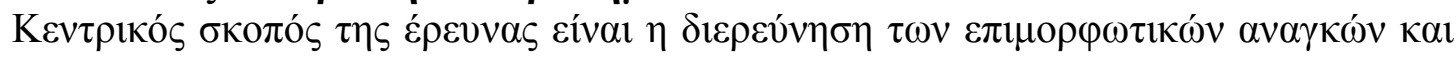

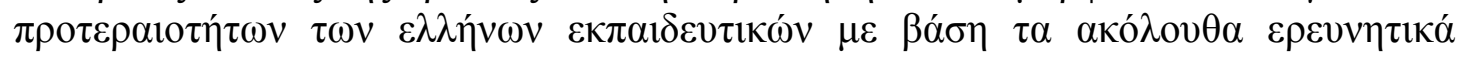
$\varepsilon \rho \omega \tau \eta \dot{\mu} \mu \alpha \alpha$ :

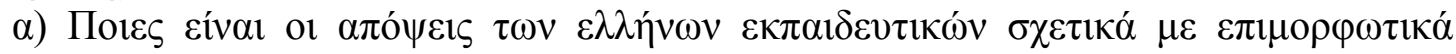

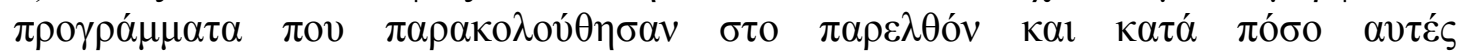

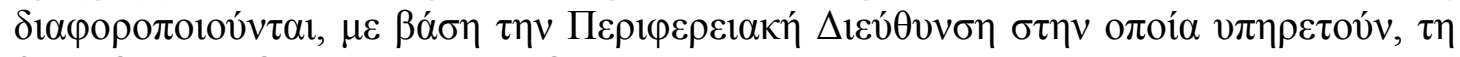

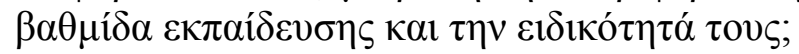

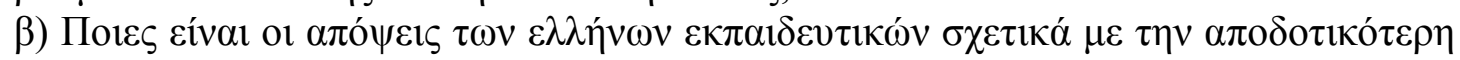

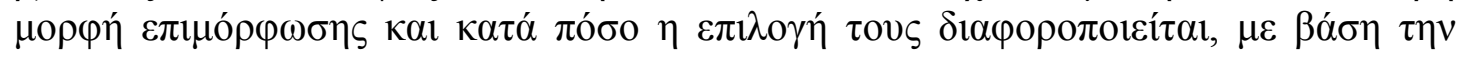

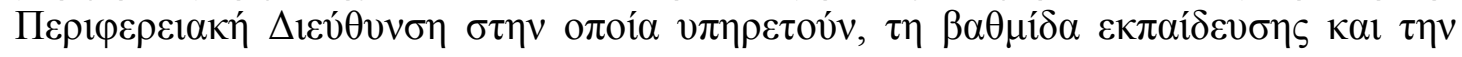

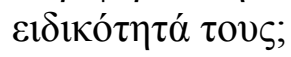

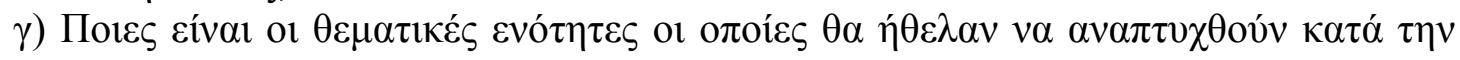

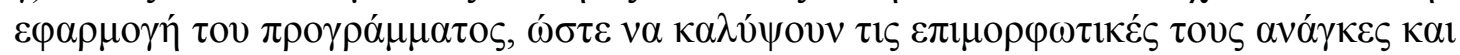

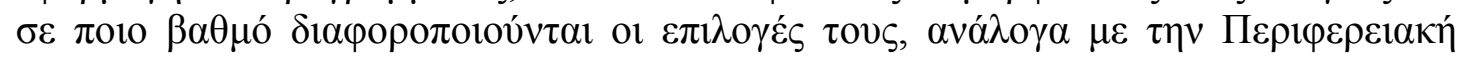

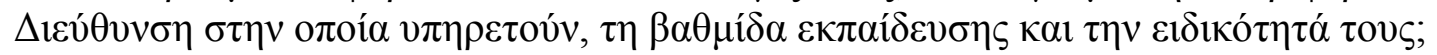

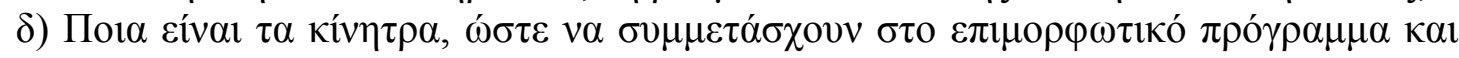

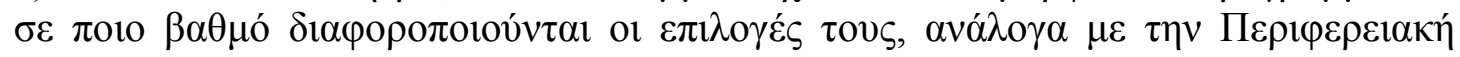

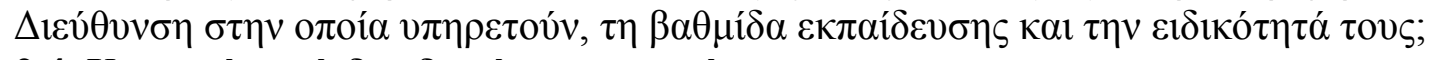

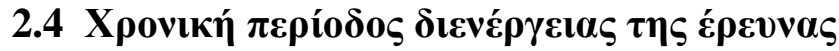

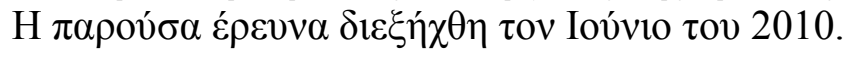

\section{$2.5 \Pi \lambda \eta \theta v \sigma \mu o ́ \varsigma \tau \eta \varsigma ~ \varepsilon ́ \rho \varepsilon v v a \varsigma$}

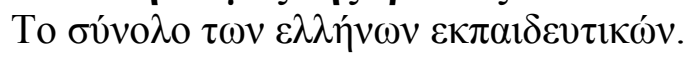

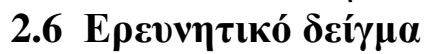

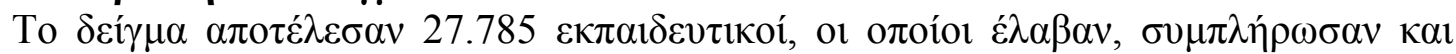

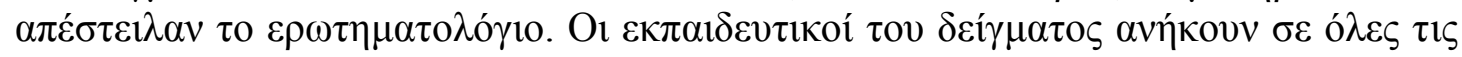

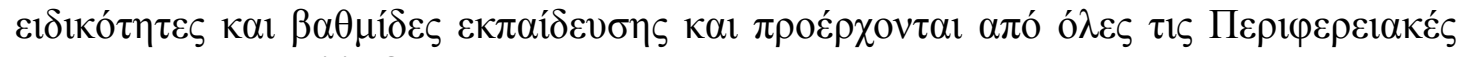

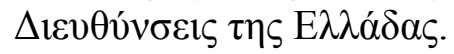

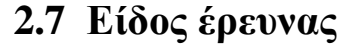

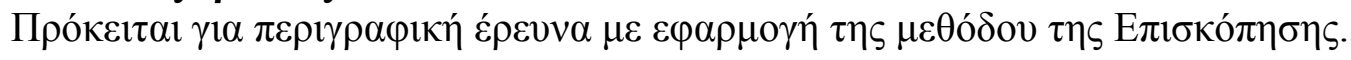

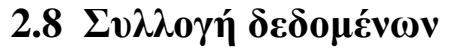




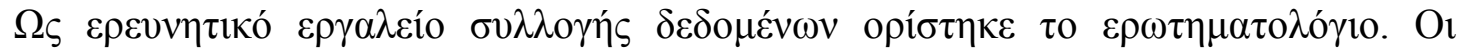

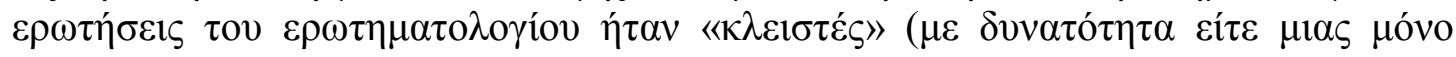

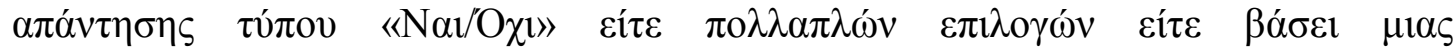

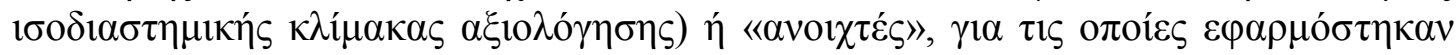

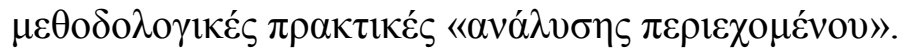

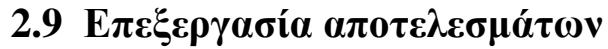

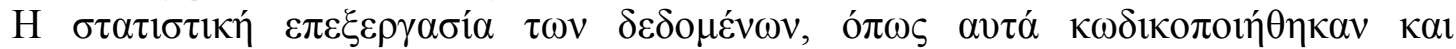

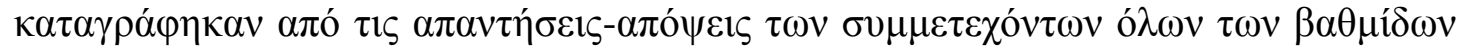

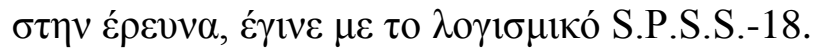

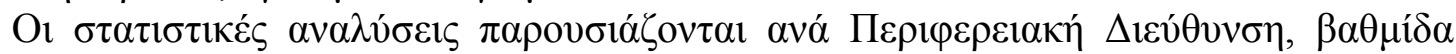

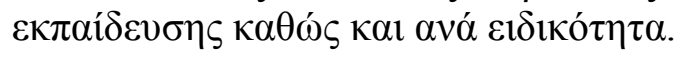

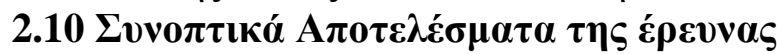

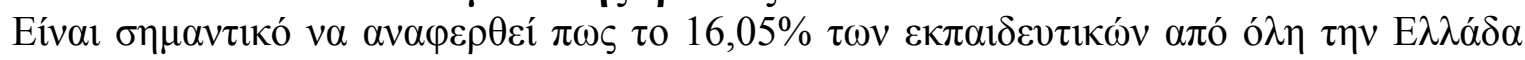

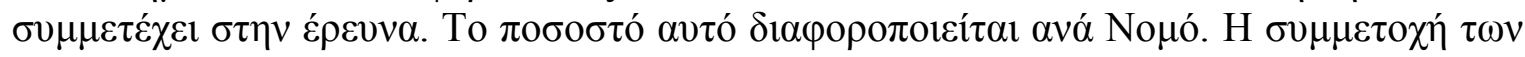

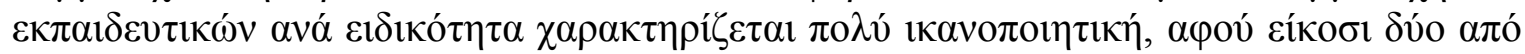

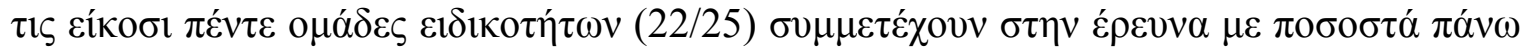

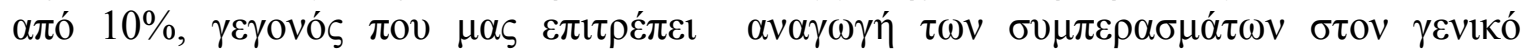
$\pi \lambda \eta \theta v \sigma \mu$ ó $^{-}$

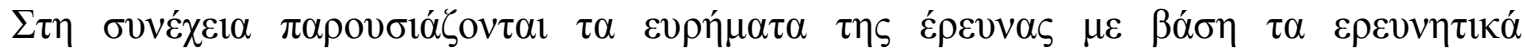

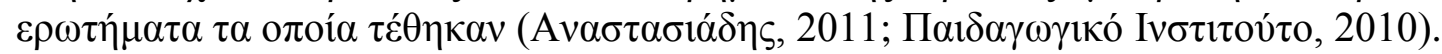

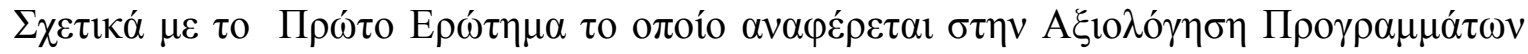

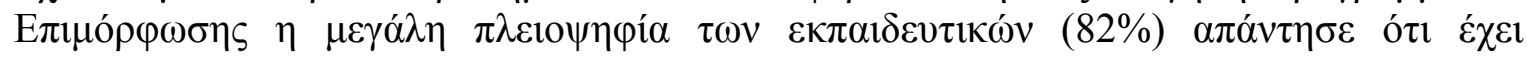

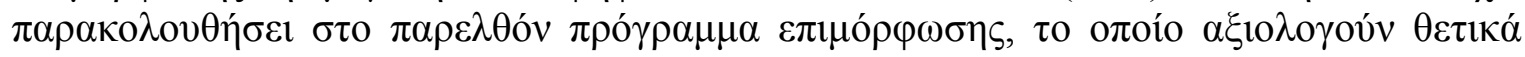

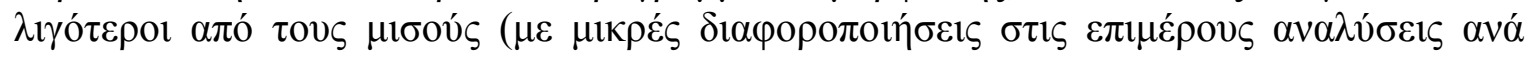

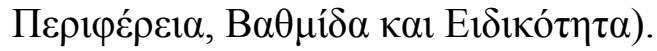

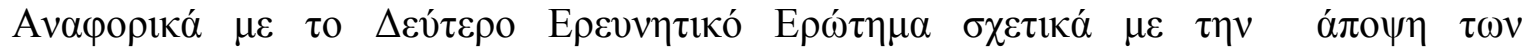

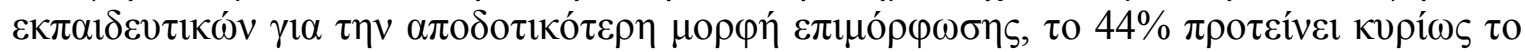

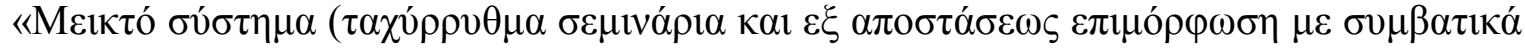

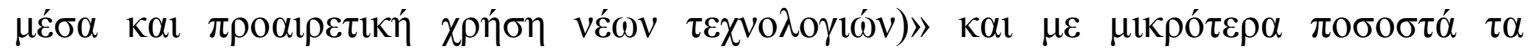

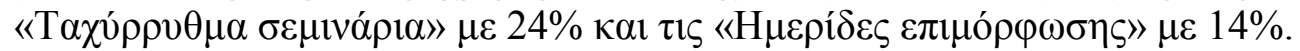

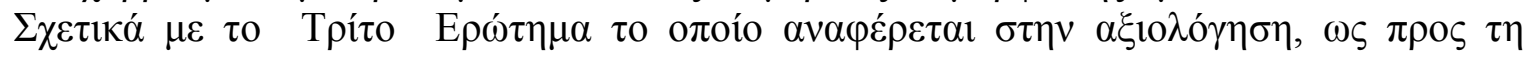

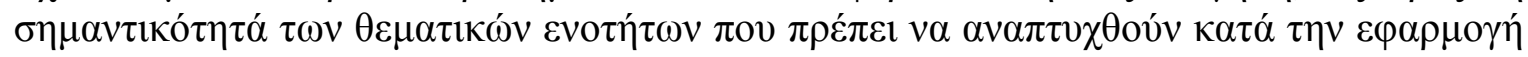

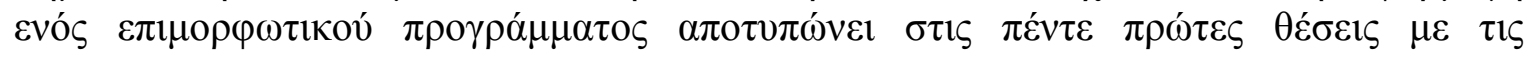

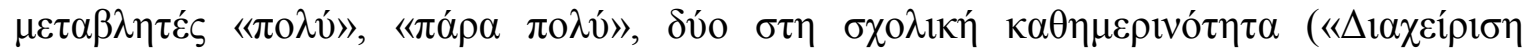

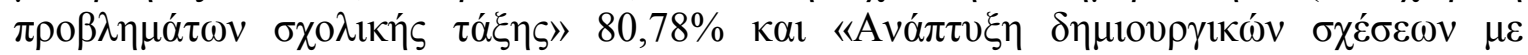

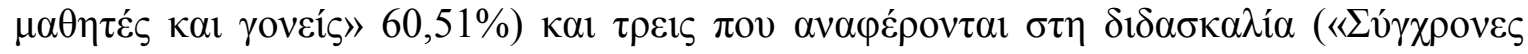

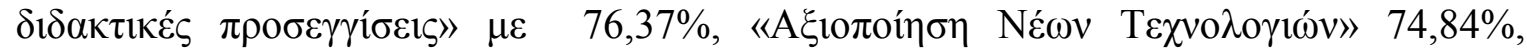

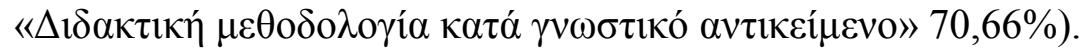

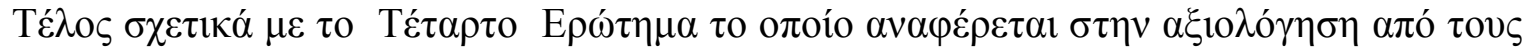

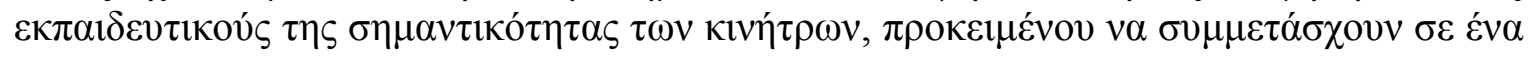

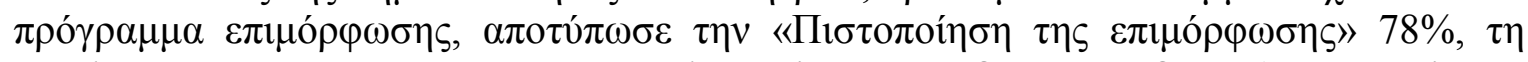

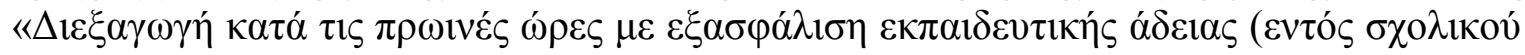

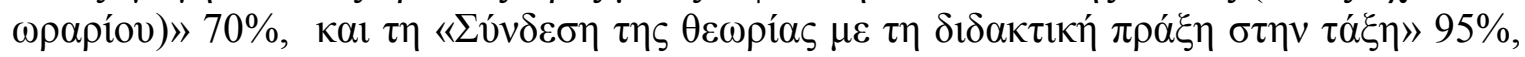

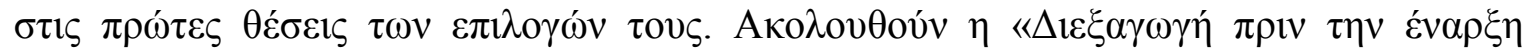

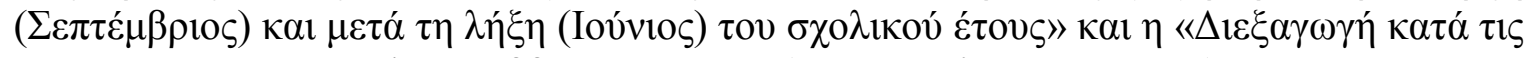

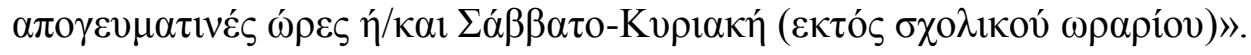

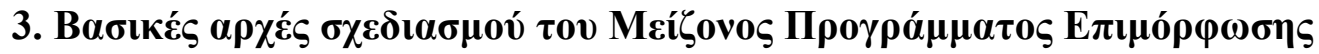 (M.П.E)}




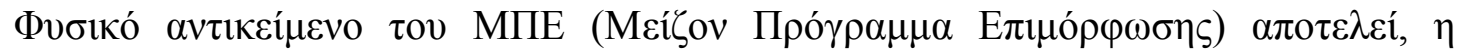

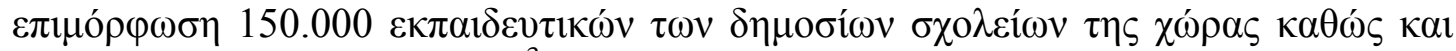

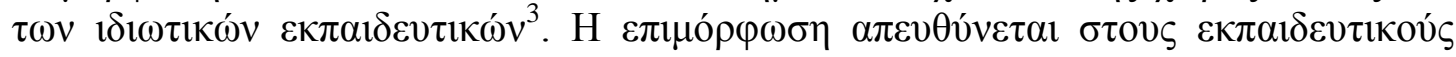

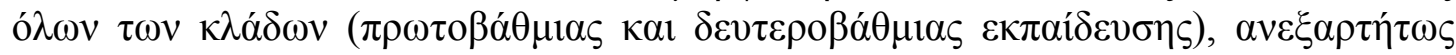

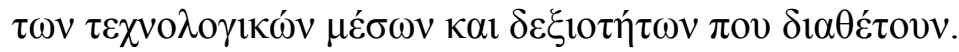

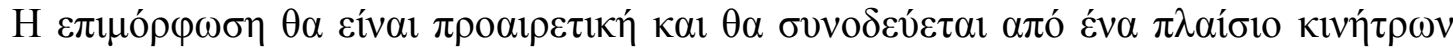

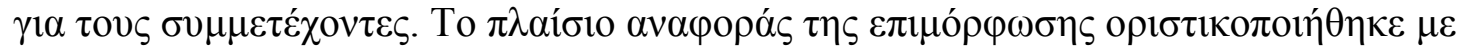

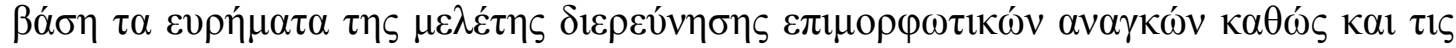

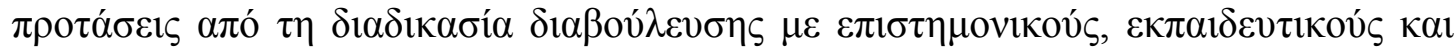

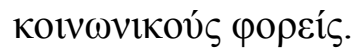

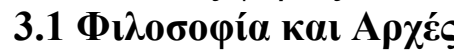

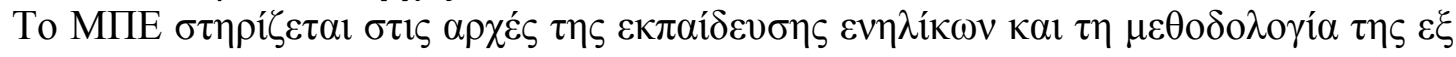

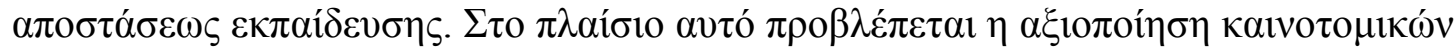

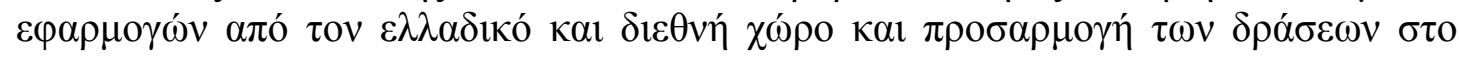

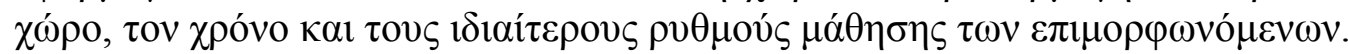

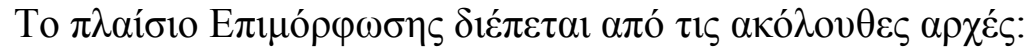

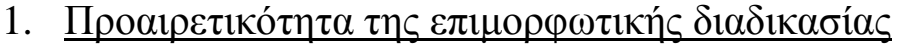

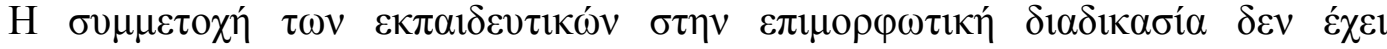

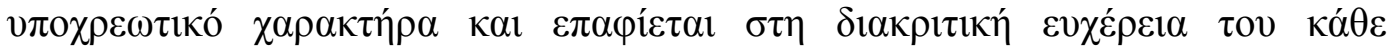

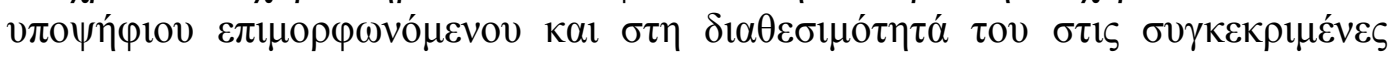

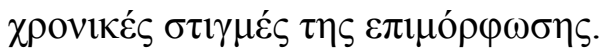

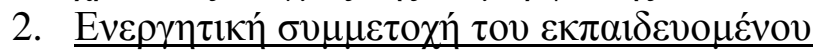

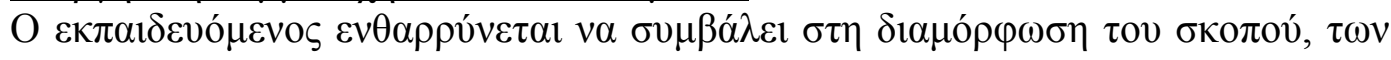

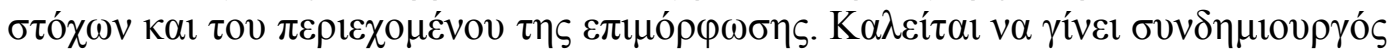

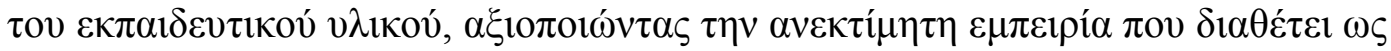

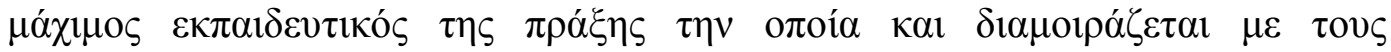

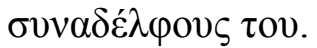

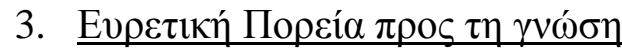

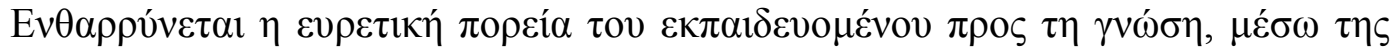

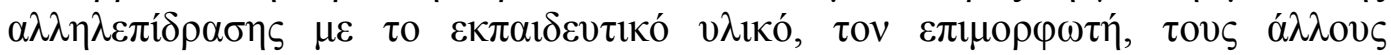

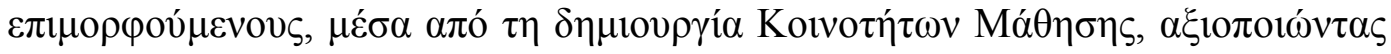

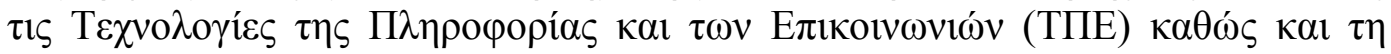

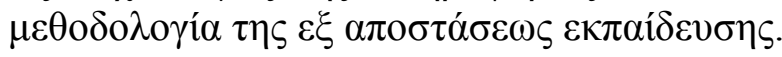

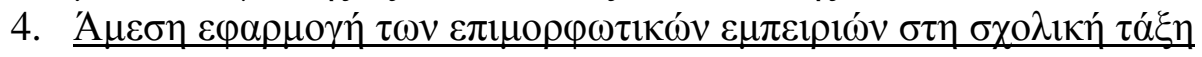

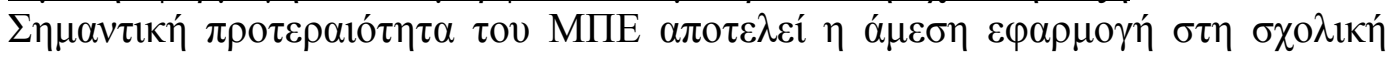

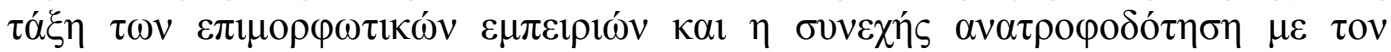

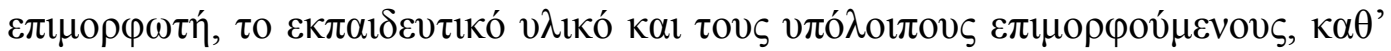

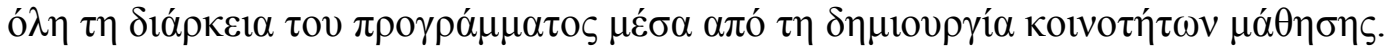

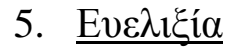

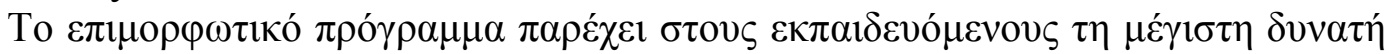

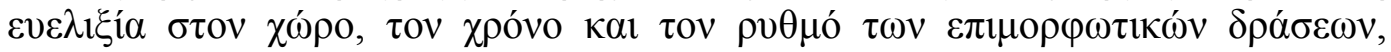

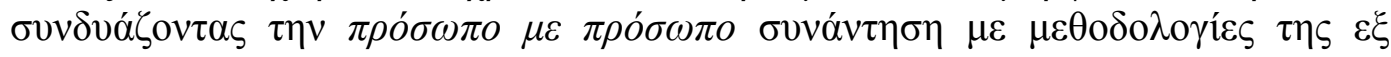

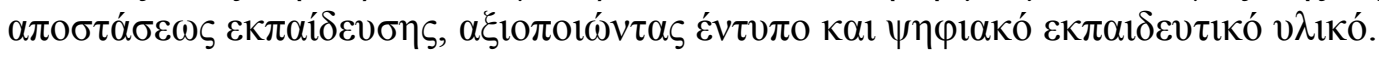

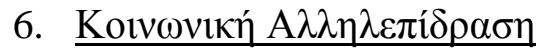

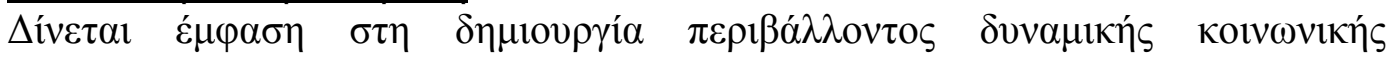

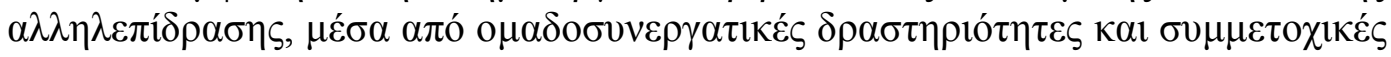
$\varepsilon \kappa \pi \alpha \imath \delta \varepsilon v \tau \imath \kappa \varepsilon ́ \varsigma \tau \varepsilon \chi \nu \imath \kappa \varepsilon ́ \varsigma$.

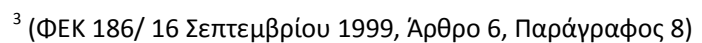




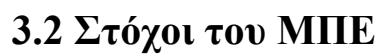

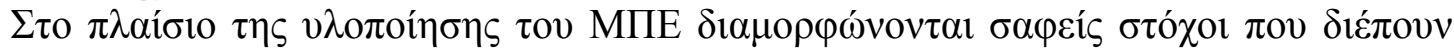

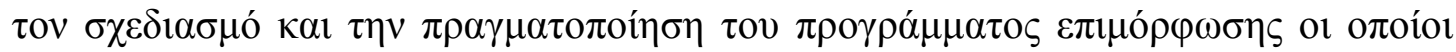

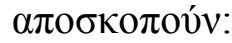

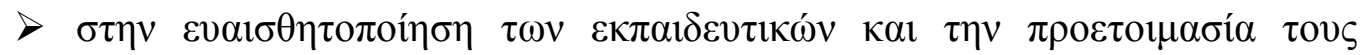

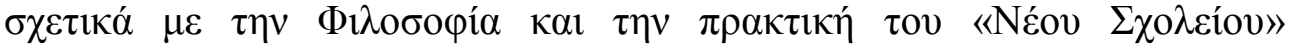

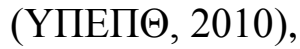

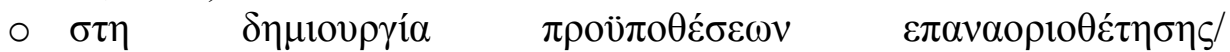

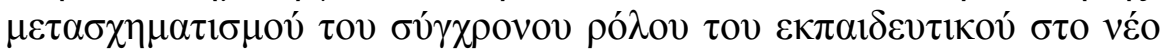

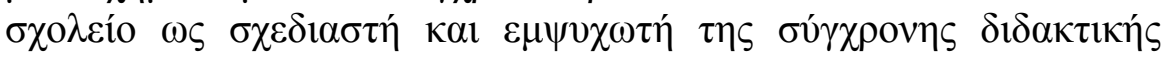

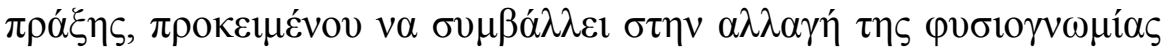

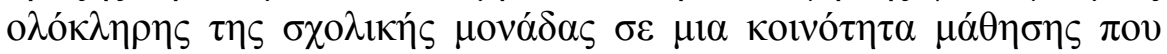

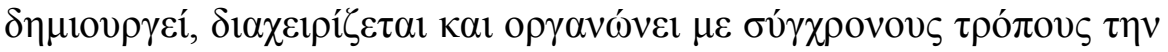
$\pi \alpha \rho \alpha \gamma \omega \gamma \eta \dot{~ \gamma \nu \omega ́ \sigma \eta \varsigma ~ \sigma \tau o v \varsigma ~ \kappa o ́ \lambda \pi o v \varsigma ~ \tau \eta \varsigma, ~}$

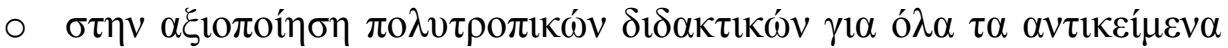

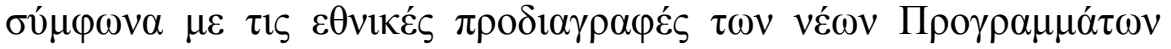
$\Sigma \pi 0 v \delta \omega \dot{v}$

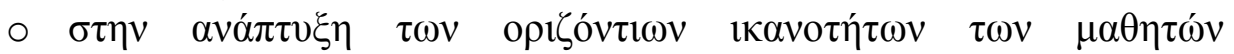

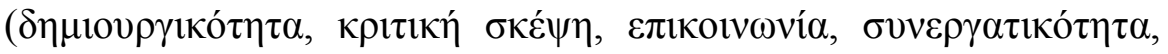

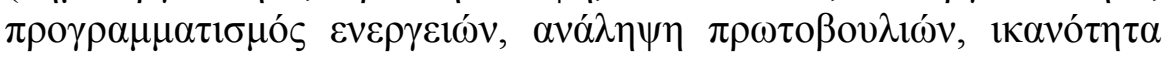
$\tau \mathrm{\tau ov}\langle\mu \alpha \theta \alpha i v \omega \pi \omega \varsigma$ v $\alpha \mu \alpha \theta \alpha i ́ v \omega »)$,

○ $\sigma \tau \eta v \alpha \xi 10 \pi \circ i ́ \eta \sigma \eta \tau \omega \nu \tau \varepsilon \chi \nu \omega ́ v ~ \sigma \tau \eta v \varepsilon \kappa \pi \alpha 1 \delta \varepsilon v \tau \imath \kappa \eta ́ ~ \delta 1 \alpha \delta ı \kappa \alpha \sigma i ́ \alpha$,

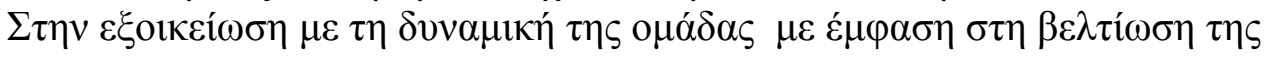

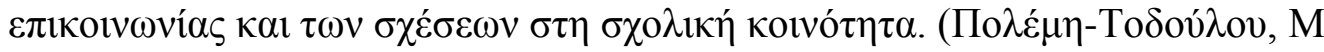

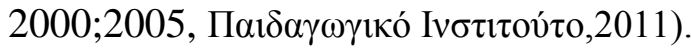

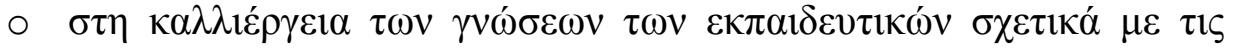

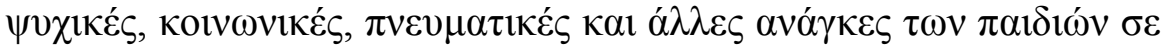

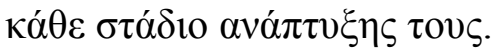

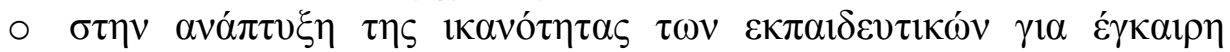

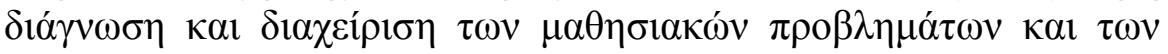

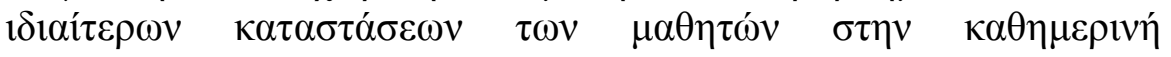

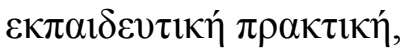

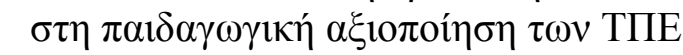

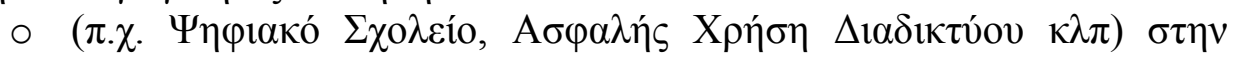

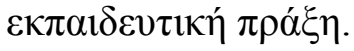

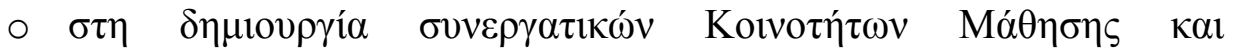

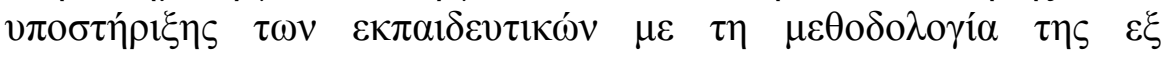

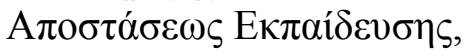

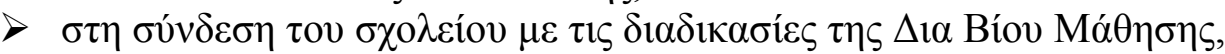

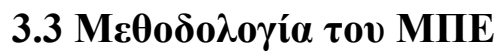

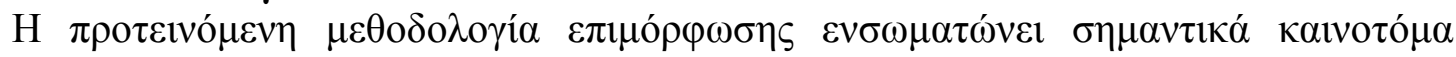

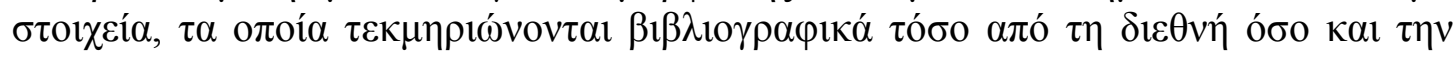

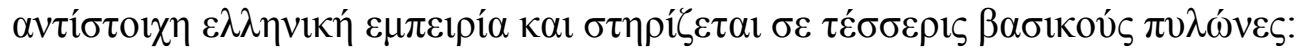

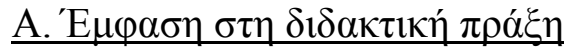

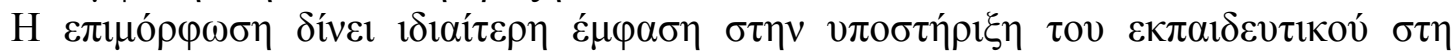

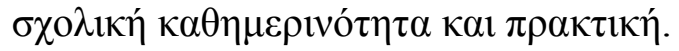

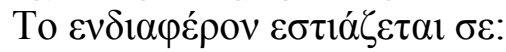




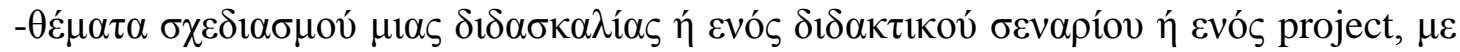

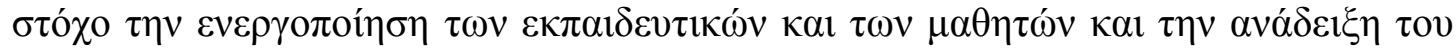

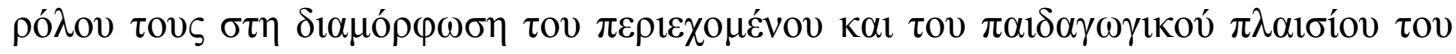
Néov $\Sigma \chi 0 \lambda \varepsilon i ́ o v$,

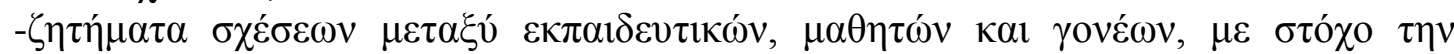

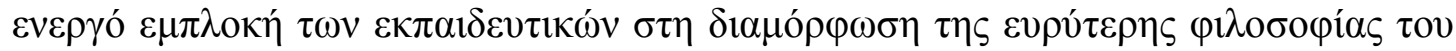

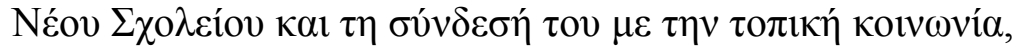

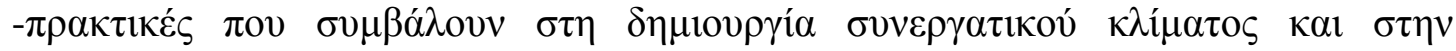

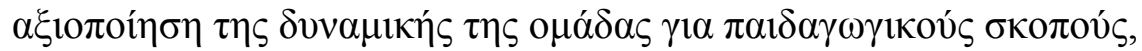

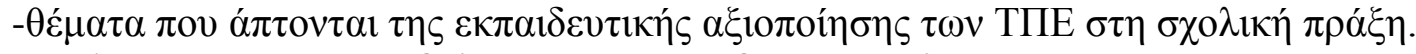

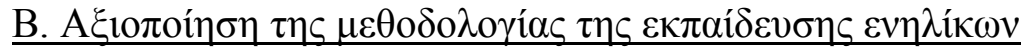

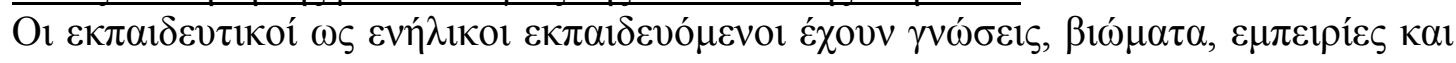

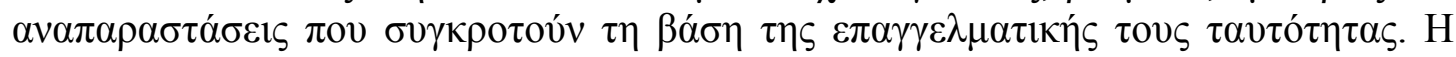

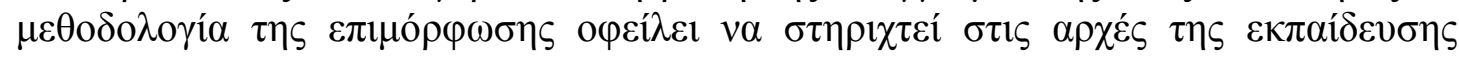

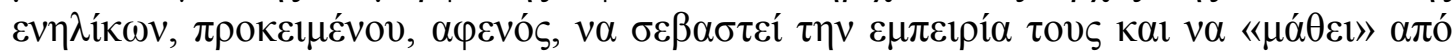

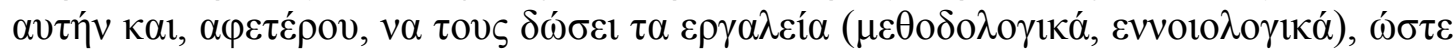

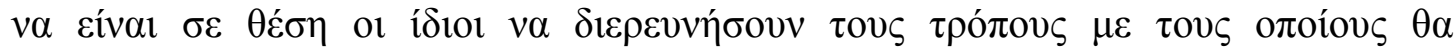

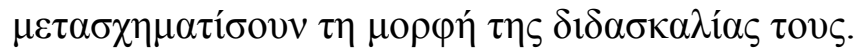

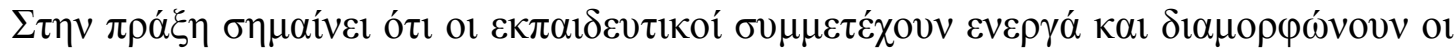

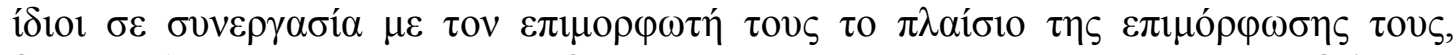

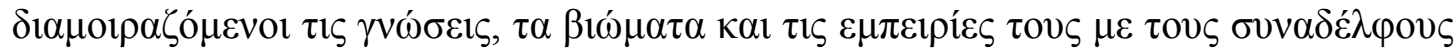

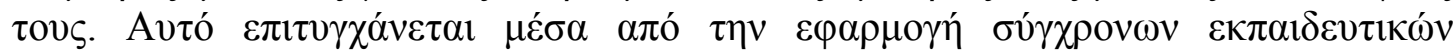

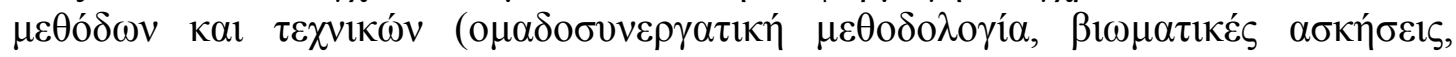

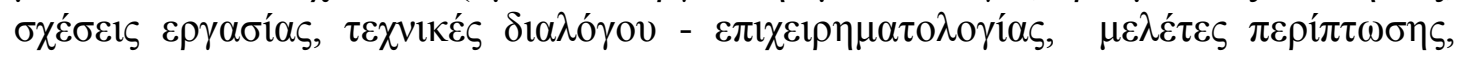

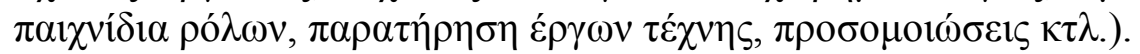

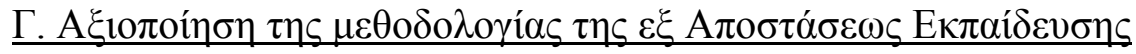

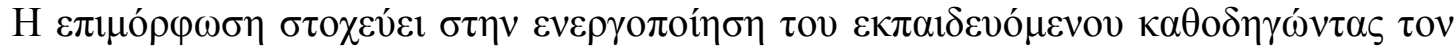

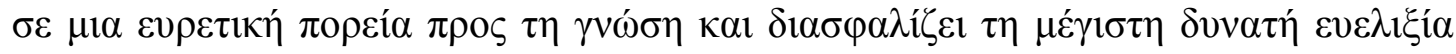

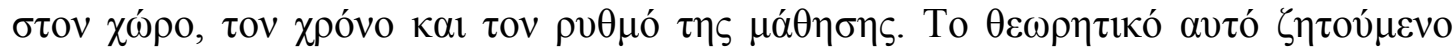

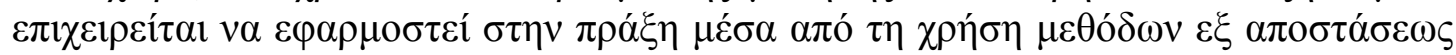

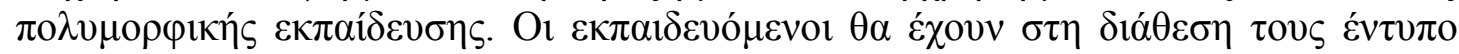

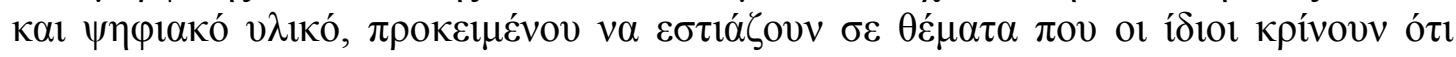

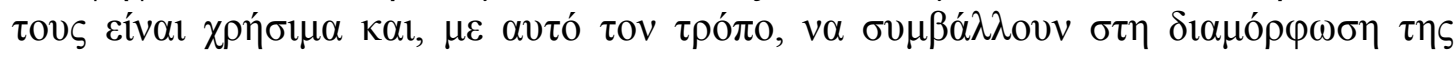

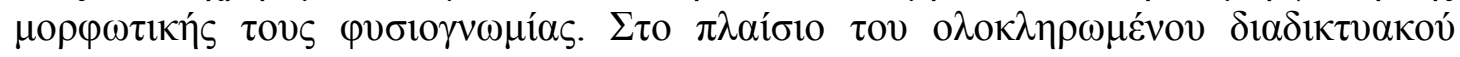

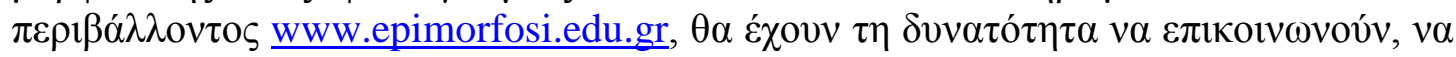

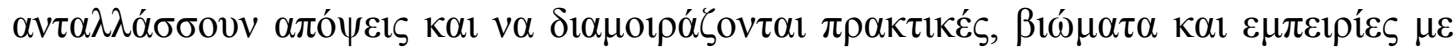

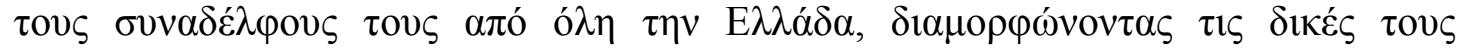

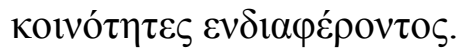

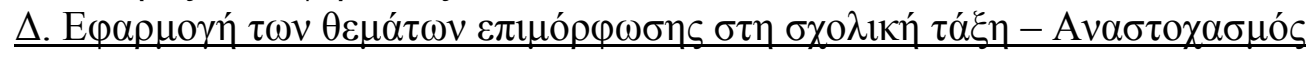

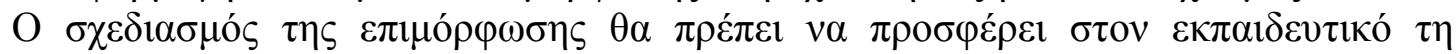

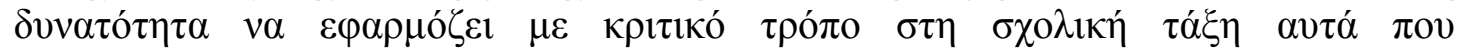

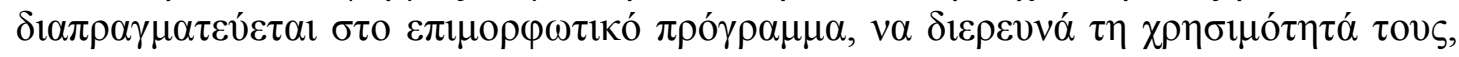

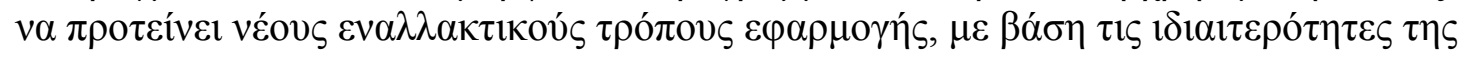

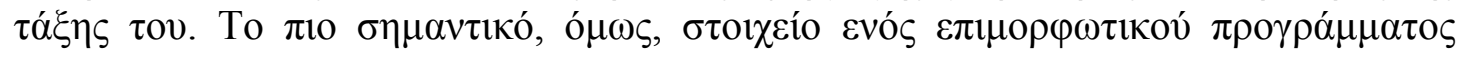

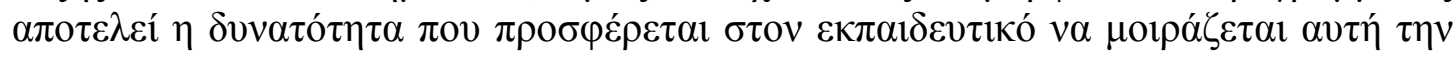

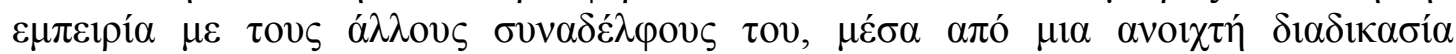

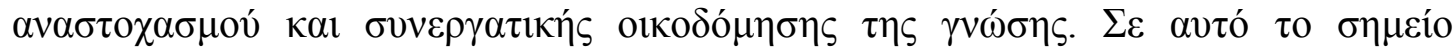

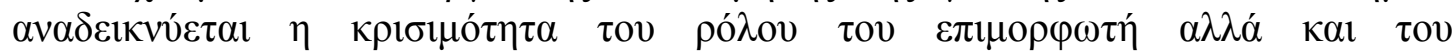

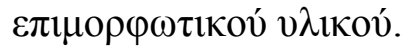




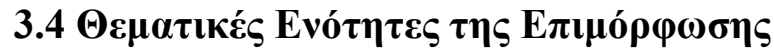

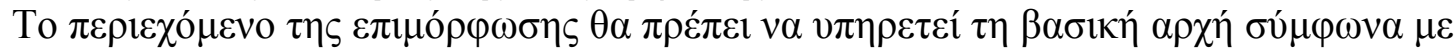

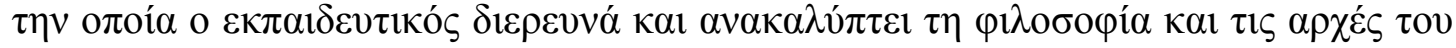

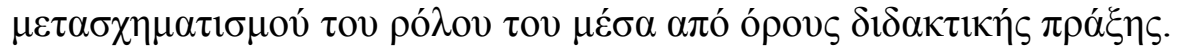

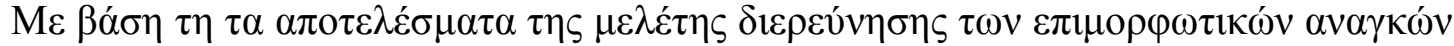

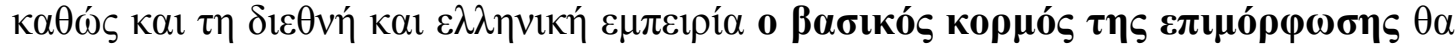
$\sigma \tau \eta \rho \imath \chi \varepsilon \dot{~} \sigma \tau 0 v \varsigma \varepsilon \xi \dot{\eta} \varsigma \pi v \lambda \omega ́ v \varepsilon \varsigma:$

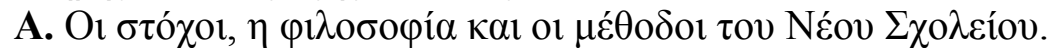

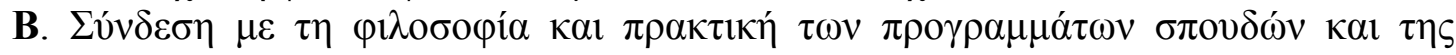

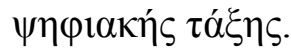

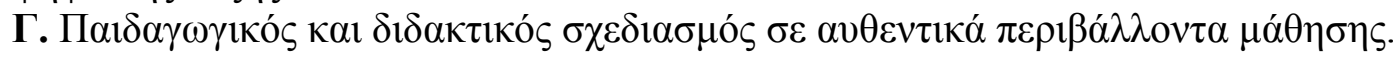

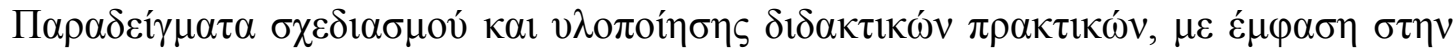

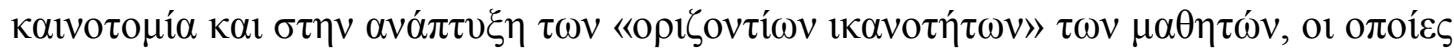

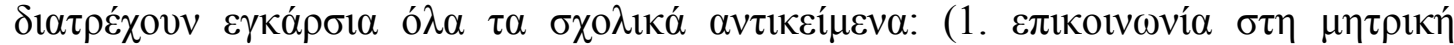

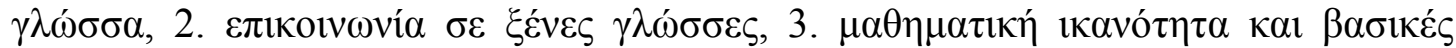

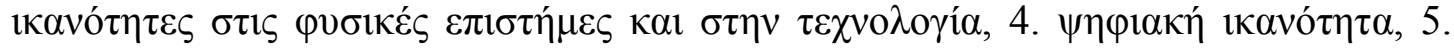

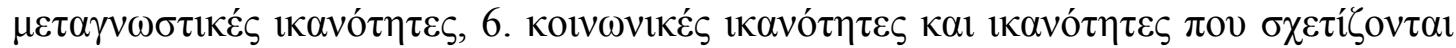

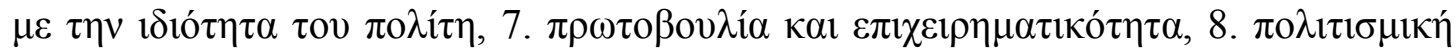

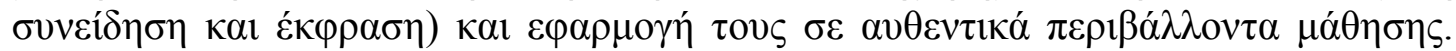

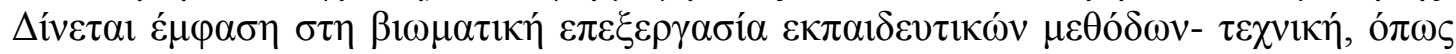

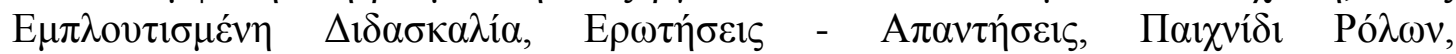
$\Delta \rho \alpha \mu \alpha \tau$ о

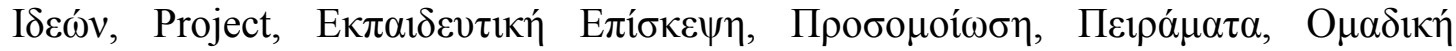

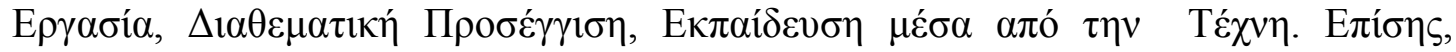

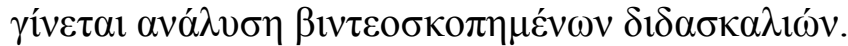

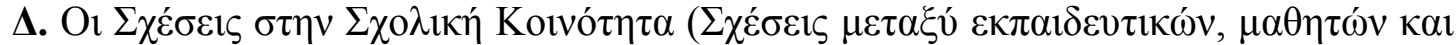

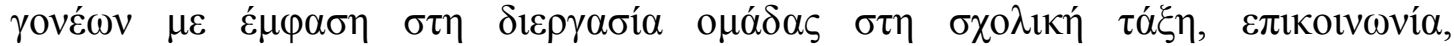

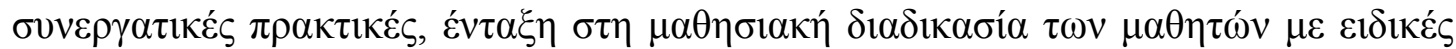

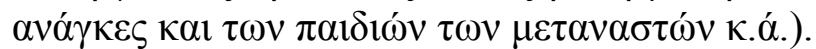

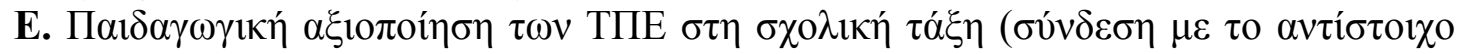
$\left.\varepsilon \pi \mu \rho \rho \varphi \omega \tau \imath \kappa o ́ ~ \pi \rho o ́ \gamma \rho \alpha \mu \mu \alpha \mathrm{B}^{\prime} \varepsilon \pi \imath \pi \varepsilon ́ \delta \circ \mathrm{)}\right)$.

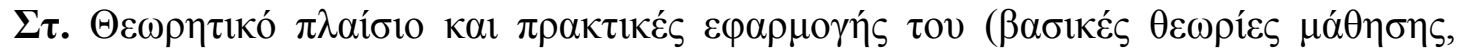

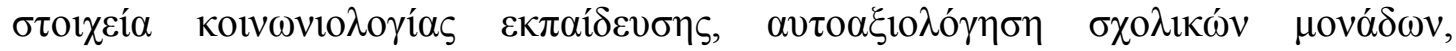

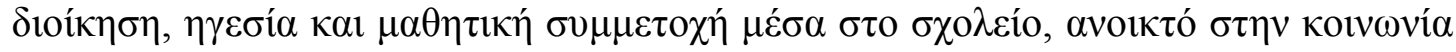

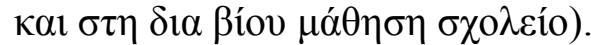

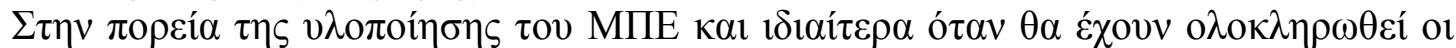

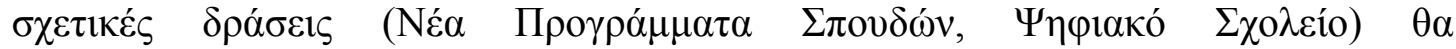

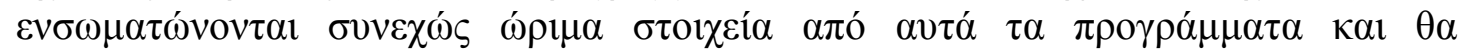

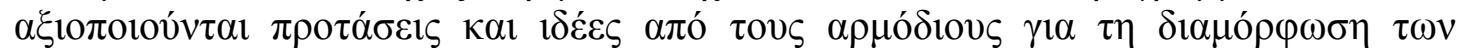

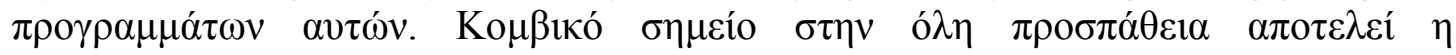

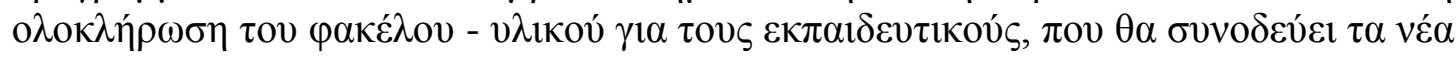
$\pi \rho о \gamma \rho \alpha ́ \mu \mu \alpha \tau \alpha \sigma \pi \circ v \delta \omega ́ v$.

\section{$3.5 \Delta \mathrm{i \alpha ́} \theta \rho \omega \sigma \eta \tau \mu \eta \mu \alpha ́ \tau \omega v$}

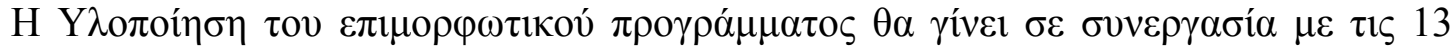

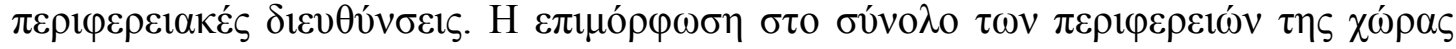

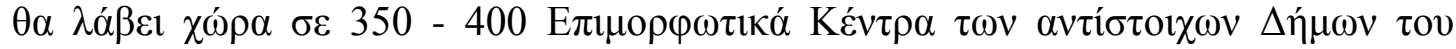




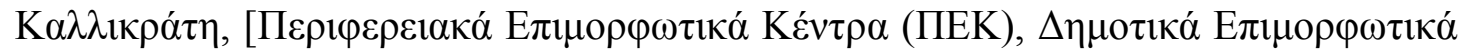

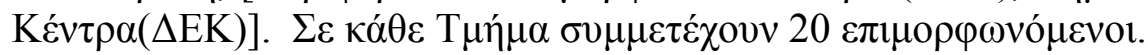

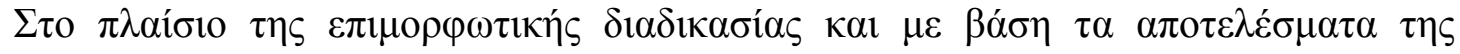

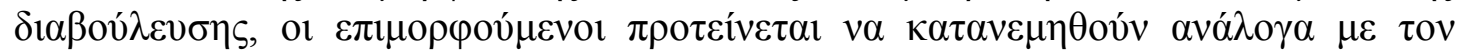

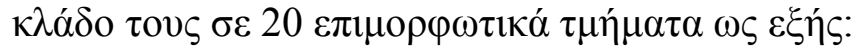

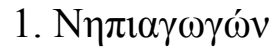

2. $\Delta \alpha \sigma \kappa \alpha ́ \lambda \omega v$

3. $\Phi$ ìo

4. $\Theta \varepsilon \circ \lambda$ ó $\gamma \omega v$

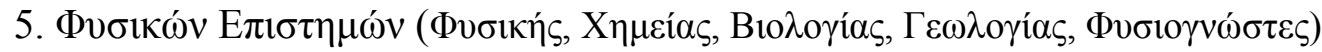

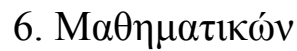

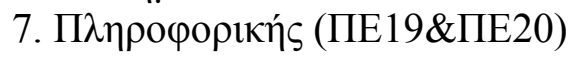

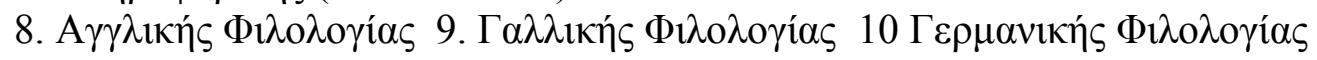

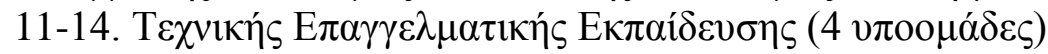

15. Фvбıкńs А $ү \omega \gamma \eta ́ s$

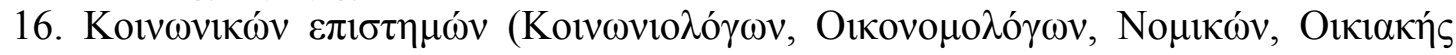
Oıкоvонías)

17. Eı̊ıќ́ А А

18. Мovбıкńs (ПЕ16/TE16)

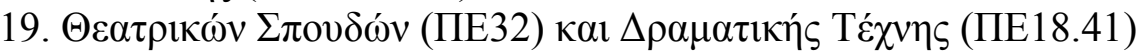

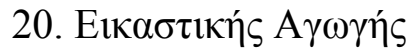

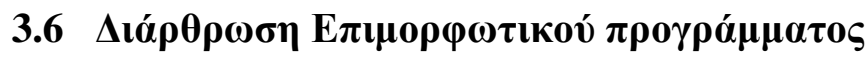

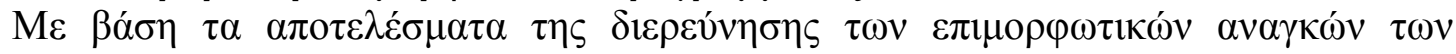

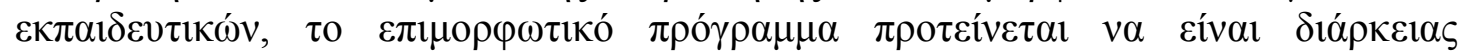

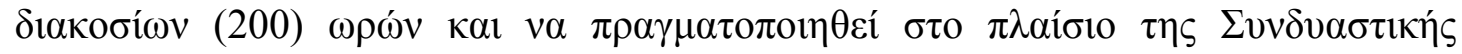

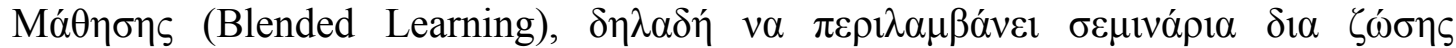

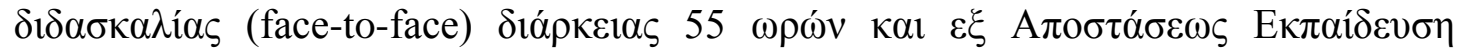

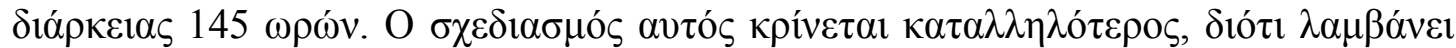

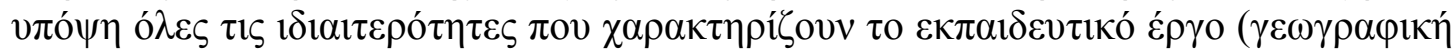

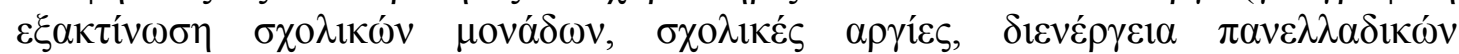

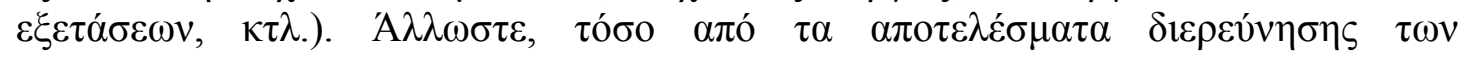

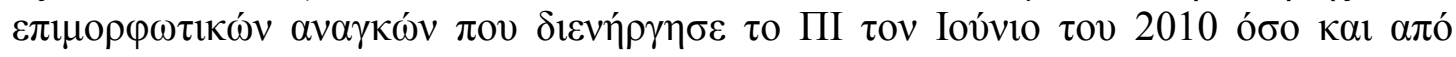

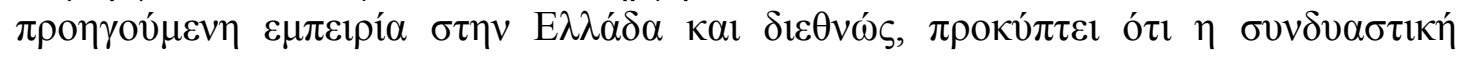

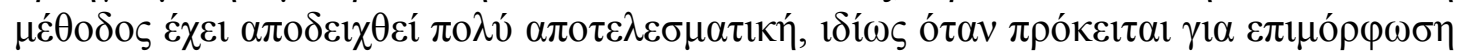

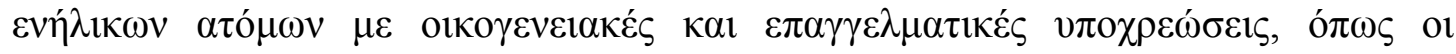

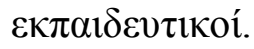

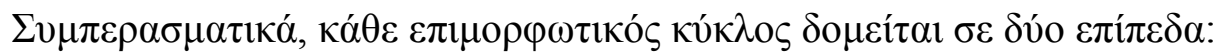

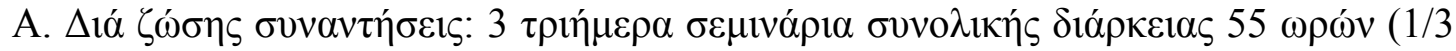

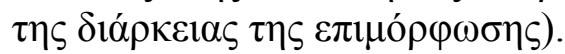

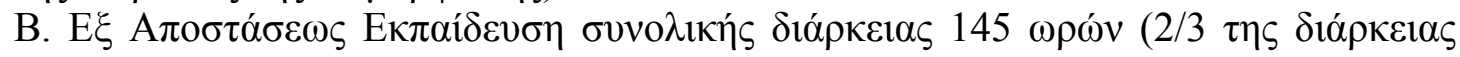
$\tau \eta \varsigma \varepsilon \pi \mu$ ó $\rho \omega \sigma \eta \varsigma)$.

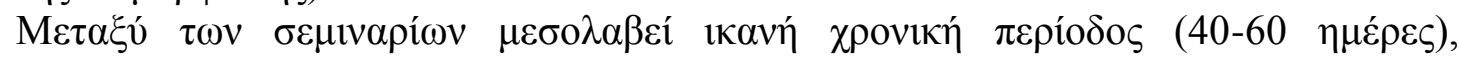

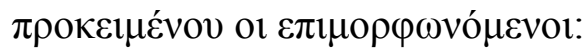

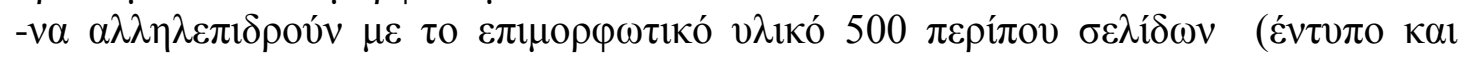
$\psi \eta \varphi 1 \alpha \kappa o ́)$,

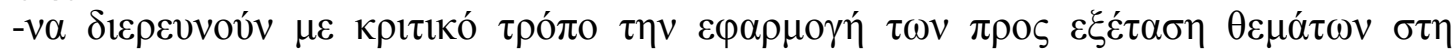

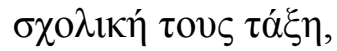

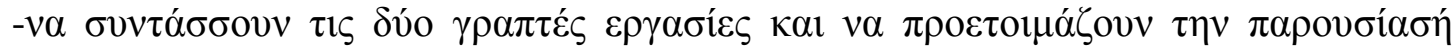
tovs. 


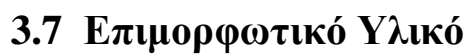

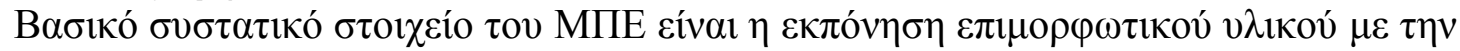

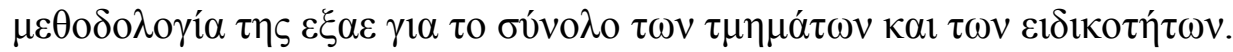

\section{$3.8 \Delta \mathrm{t \alpha \delta \iota \kappa \alpha \sigma í \alpha} \mathrm{\varepsilon \pi \imath \tau v \chi 0v́ \varsigma} \mathrm{о \lambda о \kappa \lambda \eta ́ \rho \omega \sigma \eta \varsigma} \mathrm{\varepsilon \pi ı \mu ó \rho \varphi \omega \sigma \eta \varsigma}$}

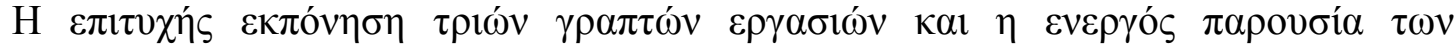

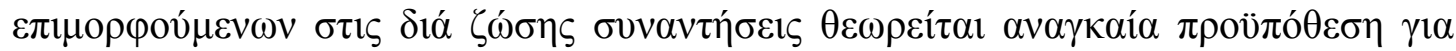

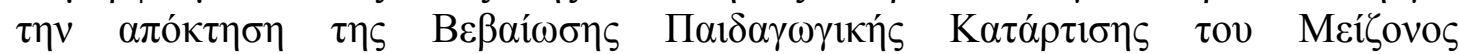

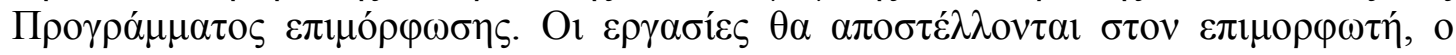

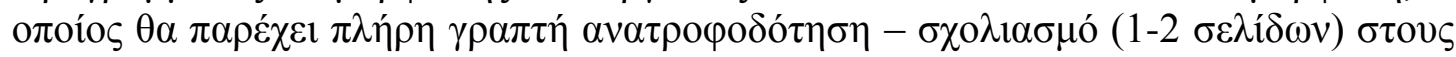
$\varepsilon \pi \iota \mu \rho \varphi \omega v o ́ \mu \varepsilon v o v \varsigma$.

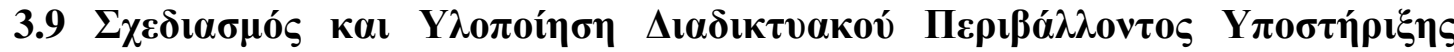

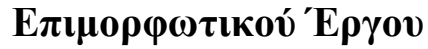

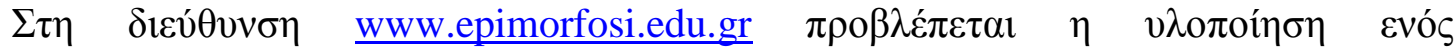

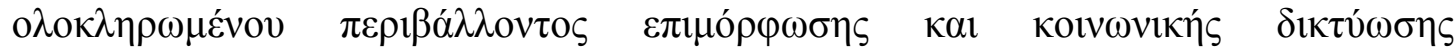

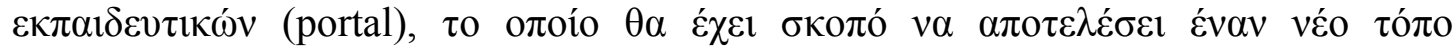

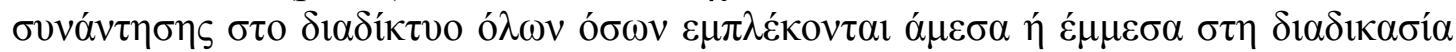

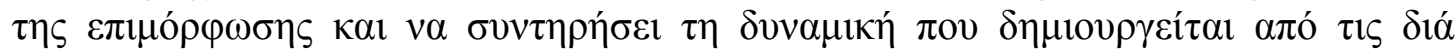

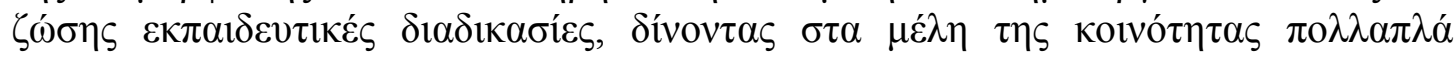

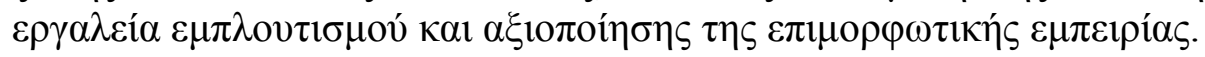

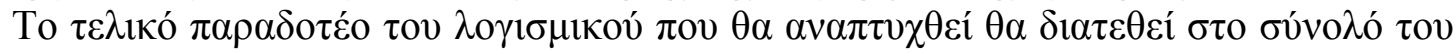

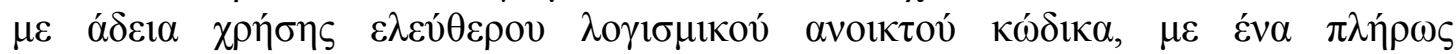

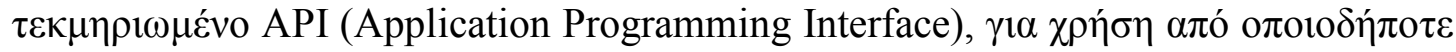

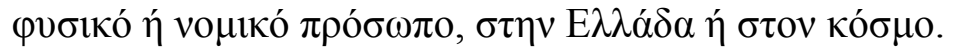

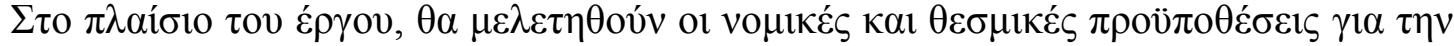

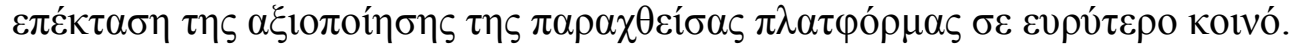

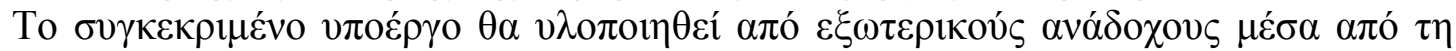

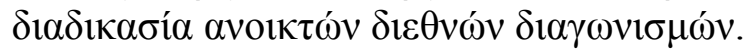

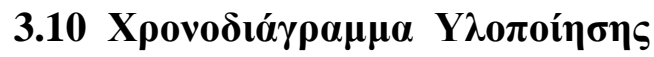

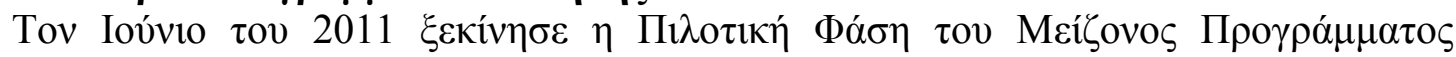

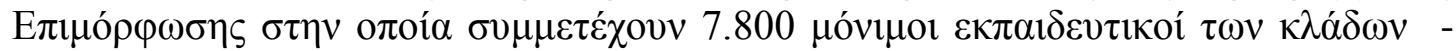

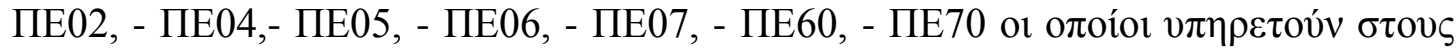

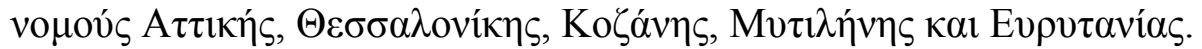

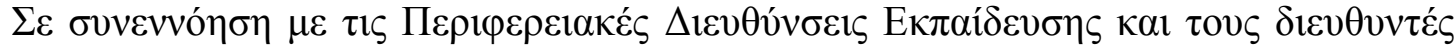

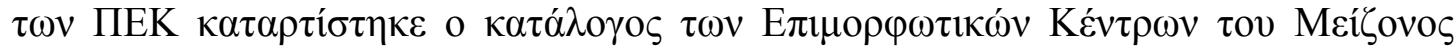

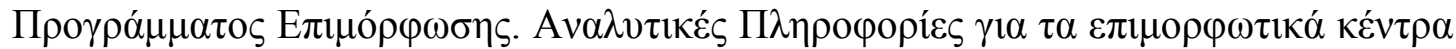

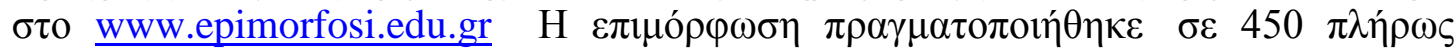

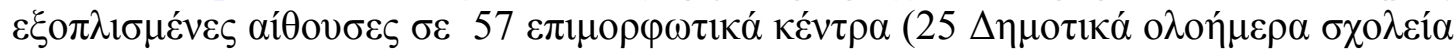

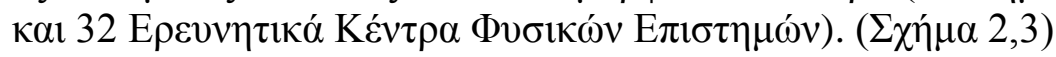




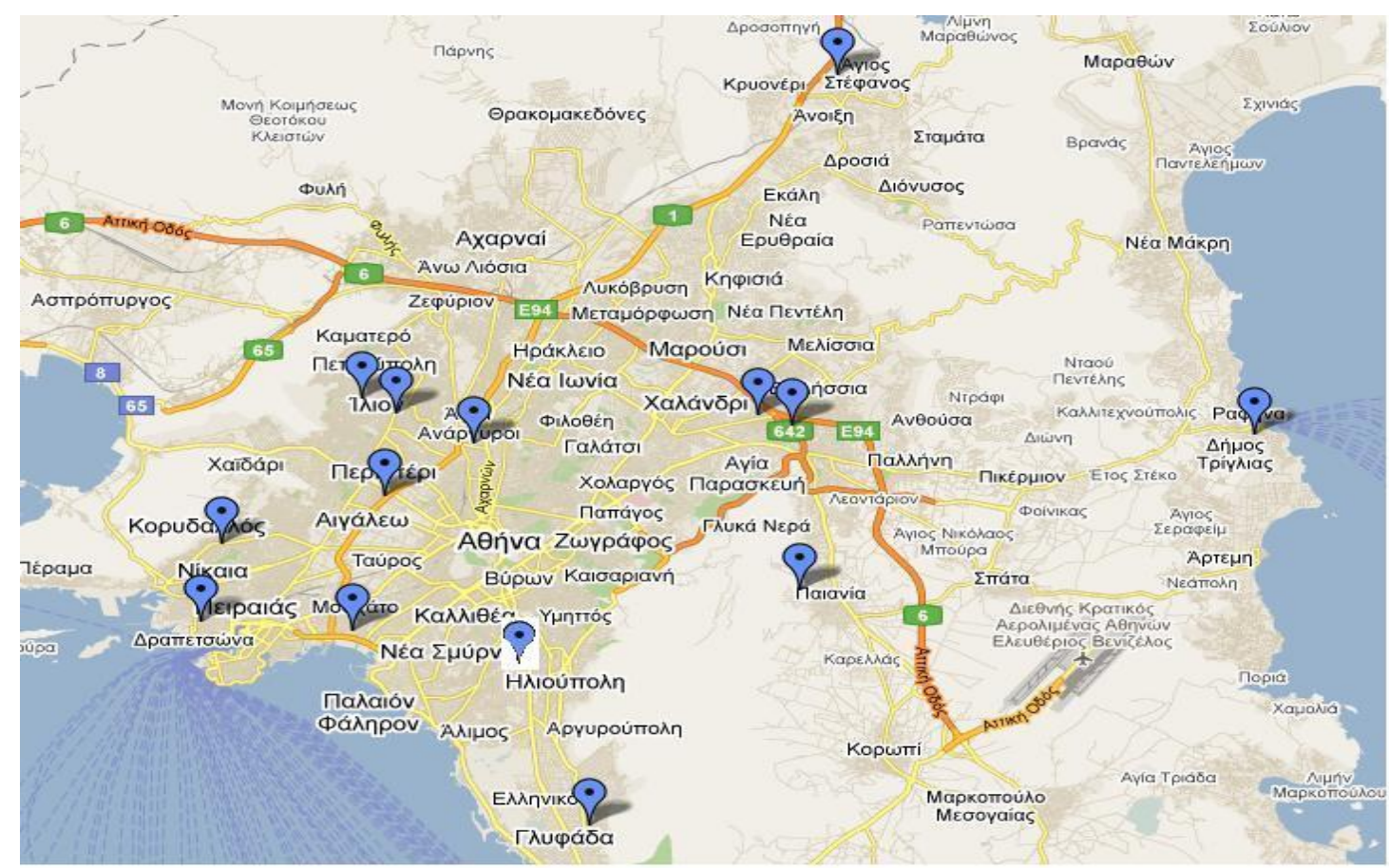

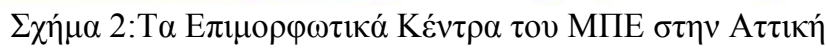

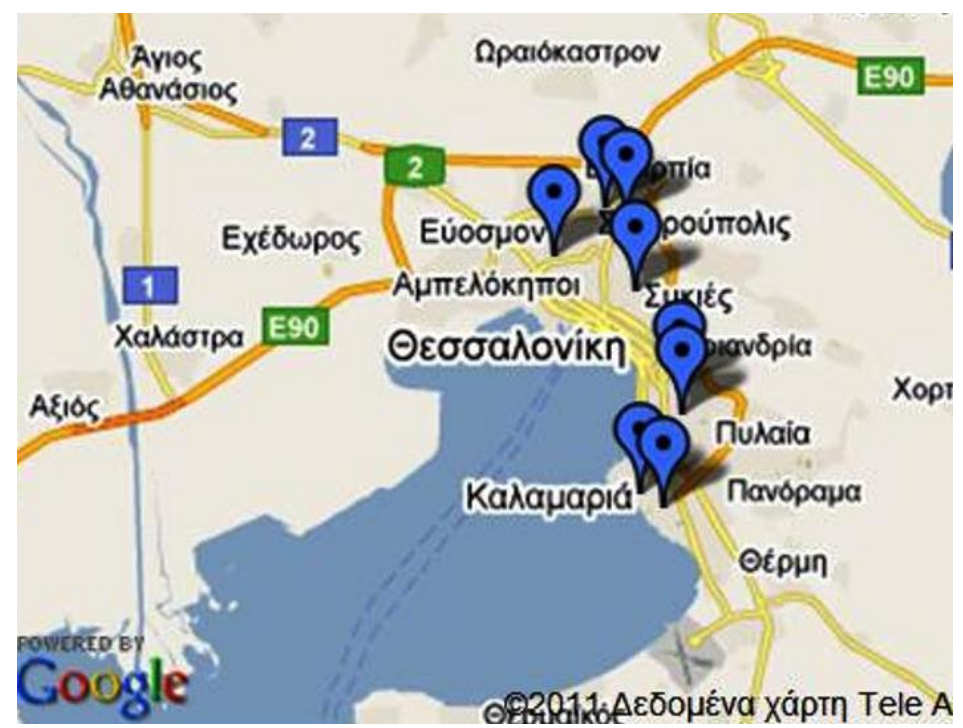

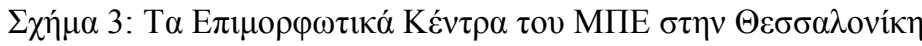

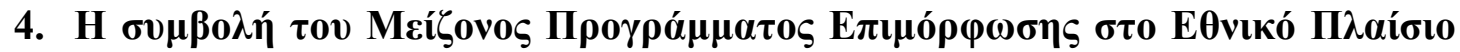

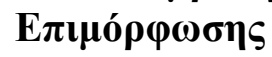

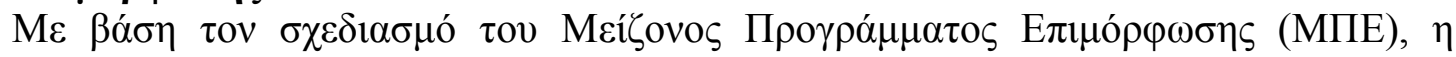

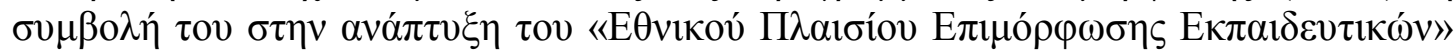

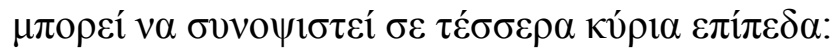

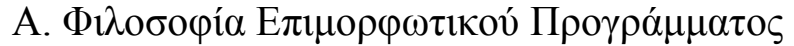

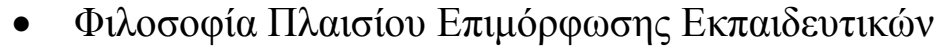

○ Eviaíos $\Sigma \chi \varepsilon \delta 1 \alpha \sigma \mu$ ó

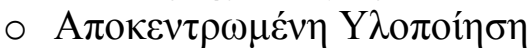

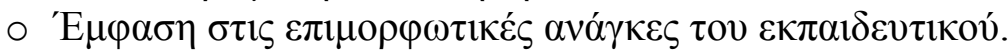

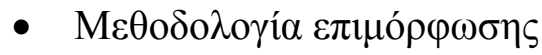


○ $\Sigma u ́ v \delta \varepsilon \sigma \eta \theta \varepsilon \omega \rho i ́ \alpha \varsigma \mu \varepsilon \tau \eta v \pi \rho \alpha ́ \xi \eta, ~ \varepsilon ́ \rho \varepsilon v v \alpha \delta \rho \alpha ́ \sigma \eta, \alpha v \alpha \sigma \tau o \chi \alpha \sigma \mu o ́ \varsigma$

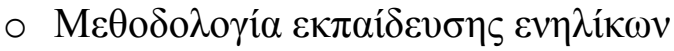

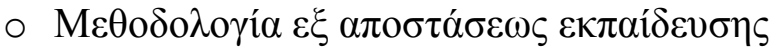

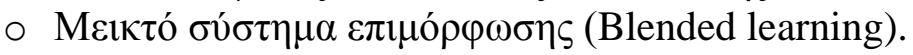

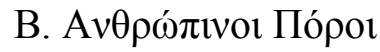

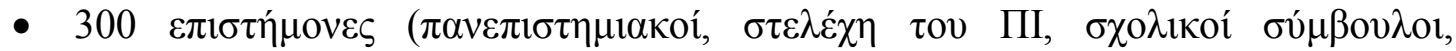

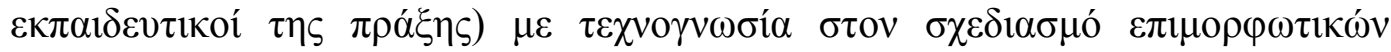

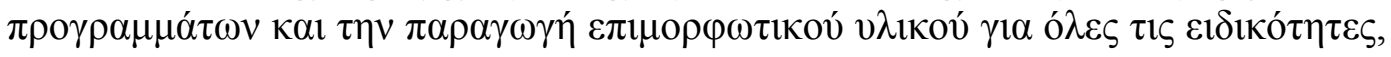

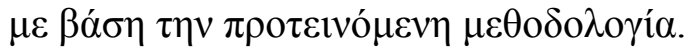

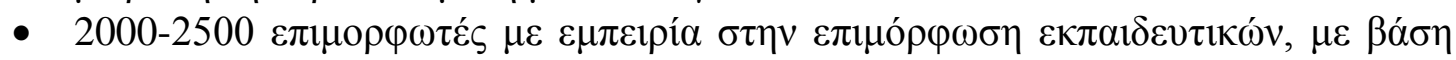

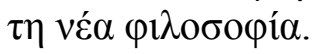

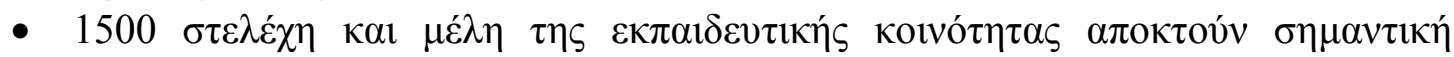

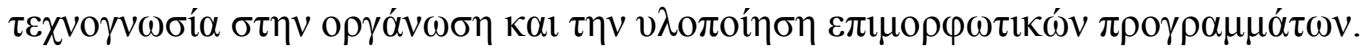

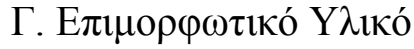

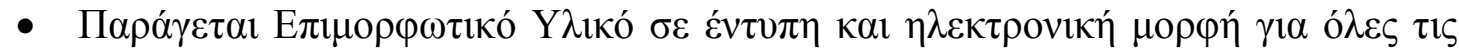

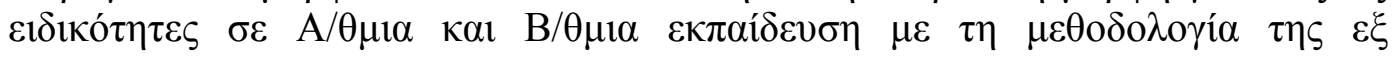

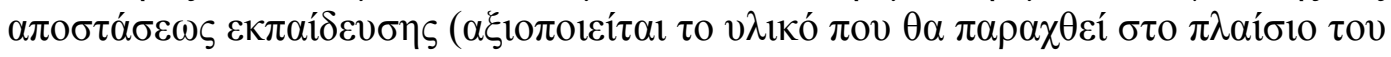

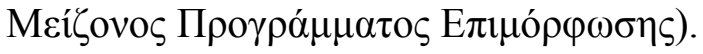

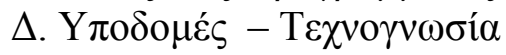

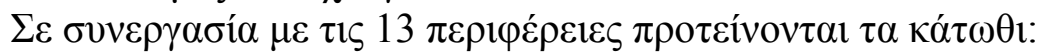

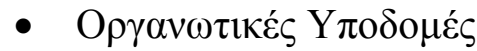

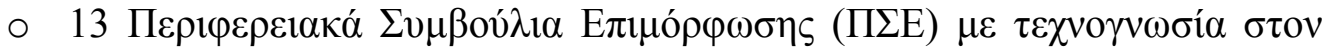

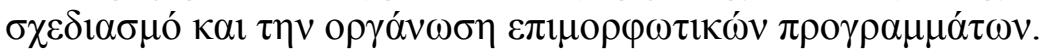

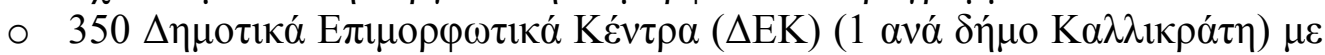

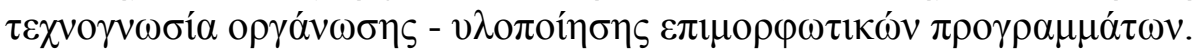

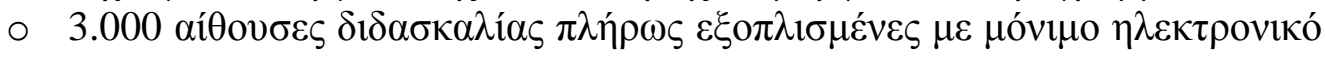

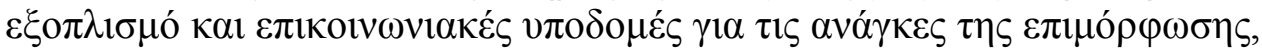

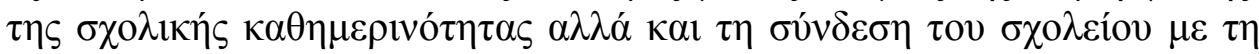

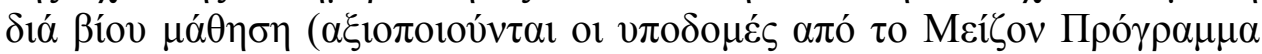

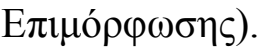

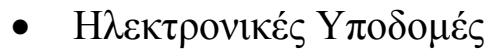

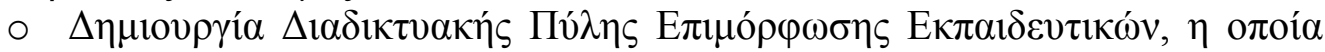

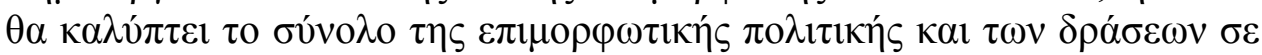

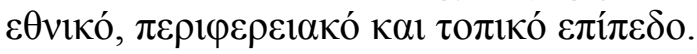

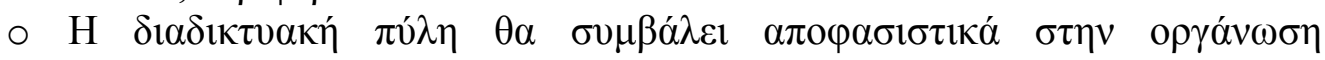

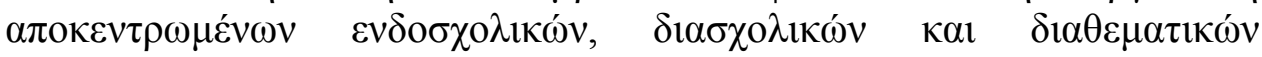

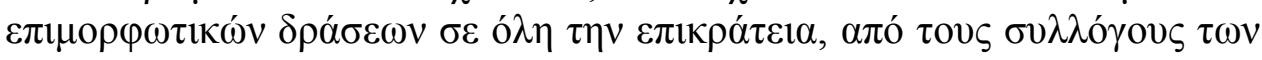

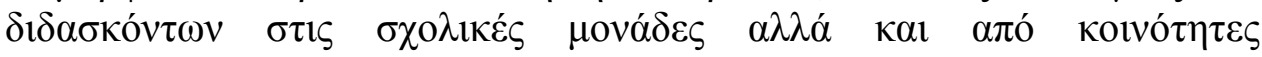
$\varepsilon \kappa \pi \alpha 1 \delta \varepsilon v \tau \imath \kappa \omega ́ v$.

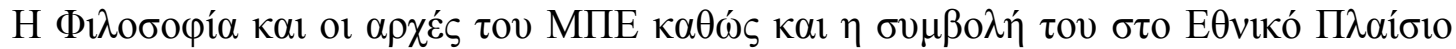

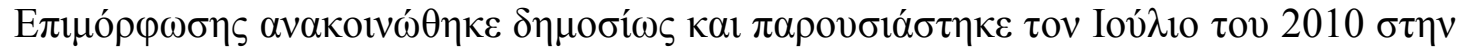

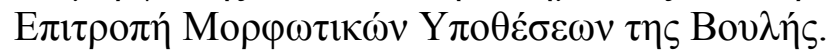

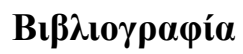

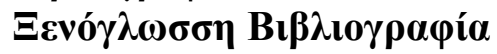

Anastasiades, P. (to be published) Design of a Blended Learning Environment for the Training of Greek teachers: Results of the survey on educational needs. In. P.Anastasiades (Editor) Blended Learning Environments for Adults: Evaluations and Frameworks, NY, IGI Global 
Anastasiades, P. (2009). Interactive Videoconferencing and Collaborative Distance Learning for K-12 Students and Teachers: Theory and Practice NY: Nova Science Publishers, Inc

Anastasiades, P., Filippousis, G., Karvunis, L., Siakas, S., Tomazinakis, A., Giza, P., Mastoraki, E. (2010). Interactive Videoconferencing for Collaborative Distance Learning in the school of 21st Century: from theory to Practice - a case study in elementary schools in Greece. Computers \& Education 54 (2010), pp. 321-339

Anastasiades, P. (2008). Blending Interactive Videoconferencing and Asynchronous Learning in Adult Education: Towards a Constructivism Pedagogical Approach. A Case Study at the University of Crete (EDIAMME). In S. Negash, M. Whitman, A. Woszczynski, K. Hoganson (Editors) Handbook of Distance Learning for Real-Time and Asynchronous Information Technology Education, NY, IGI Global

Anastasiades, P. (2007). Interactive Videoconferencing (IVC) as a Crucial Factor in Distance Education: Towards a Constructivism IVC Pedagogy Model under a cross curricular thematic approach. In E. Bailey (Ed) Focus on Distance Education Developments., NY: Nova Science Publishers, Inc

Anderson, R. (2008). Implications of the information and knowledge society for education. In J. Voogt, \&G. Knezek (Eds.), International handbook of information technology in primary and secondary education.Berlin Heidelberg New York, Springer

Cannon, T. (2007). Closing the digital divide: An assessment of urban graduate teacher education students' knowledge of information literacy and their readiness to integrateinformation literacy into their teaching. (Doctoral dissertation, University of San Francisco,2007). Retrieved from Dissertations Abstracts International database

Chinn M.D. and Fairlie,R.W (2007). The determinants of the global digital divide: a crosscountry analysis of computer and internet penetration, Oxford Economic Papers 59(2007), pp. 16-44

Cross, K.P. (1981). Adults as Learners: Increasing Participation and Facilitating learning, San Francisco, Jpssey-Bass

Darkenwald, G. \& Merriam, S. B. (1982). Adult education: Foundations of practice. Cambridge, Harper \& Row

Drucker, P.F. (1969). The Knowledge Society, New Society, 24 April, pp. 629-631

Duncombe, R., Armour, K. (2004), "Collaborative Professional Learning: From Theory to

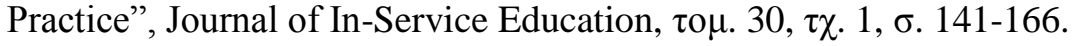

Field, J. (2000) Lifelong Learning and the New Educational Order. Stoke-on-Trent, Trentham Books

Hargreaves, A. Giles, C. (2003). The knowledge society school: An endangered entity. In Hargreaves, A. Teaching in the knowledge society: Education in the age of insecurity. Maidenhead and Philadelphia, Open University Press

Jarvis, P. (Ed.) (2001). The Age of Learning: education and the knowledge society. London, Kogan Page

Kozma, R. B. (2001). Counterpoint theory of learning with media. In R. E. Clark (Ed.), Learning from media: Arguments, analysis, and evidence, pp.137-178, Greenwich, CT: Information Age Publishing Inc

Kozma, R. B. (Ed.). (2003). Technology, innovation and educational change: A global perspective. Eugene(OR): International Society of technology in Education

Levine, T. \& Donitsa - Schmidt, S. (1998). Computer use, confidence, attitudes, and Knowledge: a casual analysis, Computers in human Behavior, 14, pp. 125-146

Mikropoulos, T. A. \& Bellou, J. (2010). The Unique Features of Educational Virtual Environments, In C. M. Stewart, C. C. Schifter \& M. E. Markaridian Selverian (Eds.) Teaching and Learning with Technology, pp. 249-258, Routledge

Miller, H. (1967). Participation of adults in education: A force-field analysis. Boston: Boston University Center for the Study of Liberal Education for Adults

National Foundation for the Improvement of Education (1996). Teachers Take Charge of Their Learning. Transforming Professional Development for Student Success, Washington D.C, National Foundation for the Improvement of Education 
Paulsen. M., (2003). Online education and learning management systems. Global e-learning in a Scandinavian perspective. Oslo: NKI Forlaget. pp 337

Rubenson, K. (1977). Participation in Recurrent Education: A Research Review, Paris,Center for Educational Research and Innovation, Organization for Economic Cooperationand Development

Rubenson, K., \& Schuetze, H. G. (2000). Lifelong learning for the knowledge society: Demand, supply,and policy dilemmas. In K. Rubenson \& H. G. Schuetze (Eds.), Transition to the knowledge society: Policies and strategies for individual participation and learning. Vancouver: UBC (Institute for European Studies)

Voogt, J., \& Pelgrum H. (2005). ICT and curriculum change. Human Technology: An Interdisciplinary Journal on Humans in ICT Environments, 1(2), 157-175

Webster, F. (2002). Theories of the information society (2nd ed.). London, Routledge

Woodrow J. E. J. (1994). The development of computer-related attitudes of secondary students, Journal of Computing Research, 11, 307-338

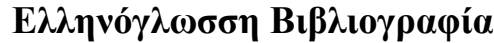

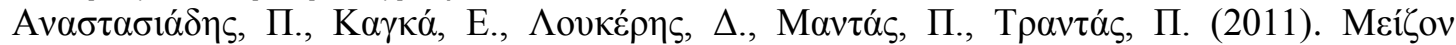

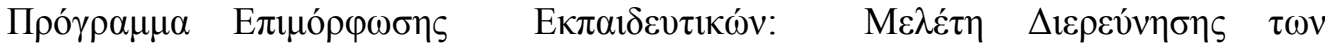

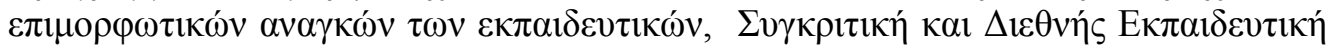

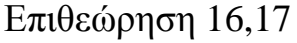

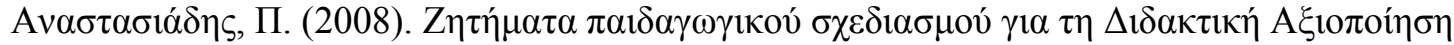

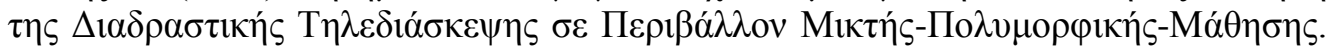

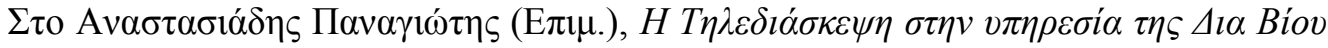

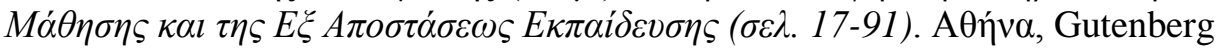

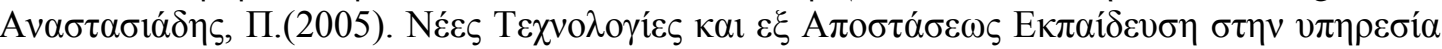

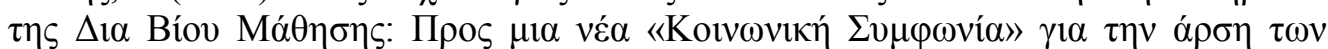

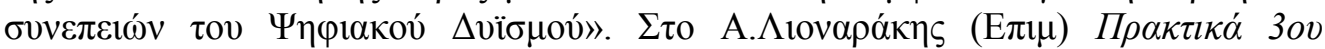

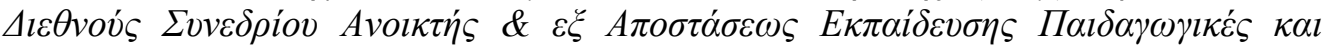

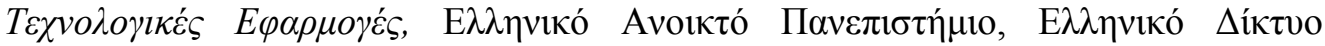

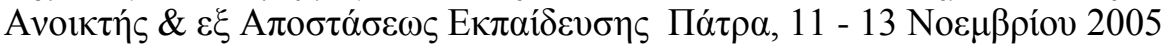

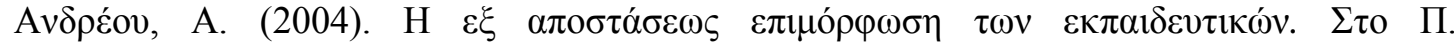

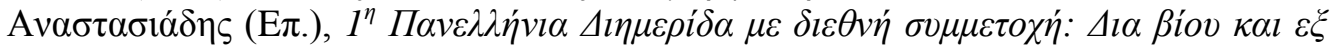

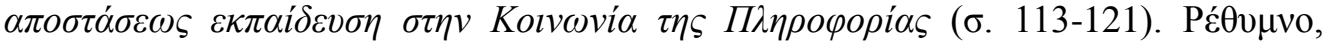

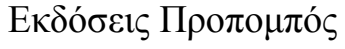

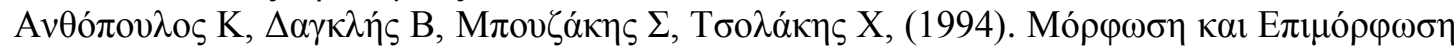

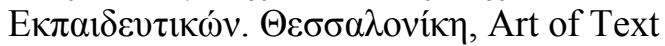

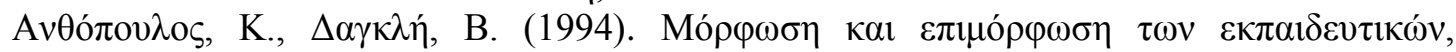
$\Theta \varepsilon \sigma \sigma \alpha \lambda \mathrm{oví \kappa \eta}$

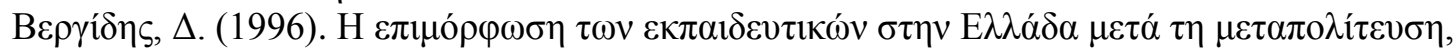

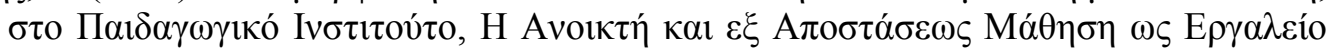

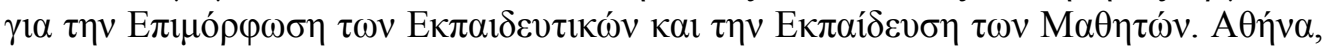
П.I.

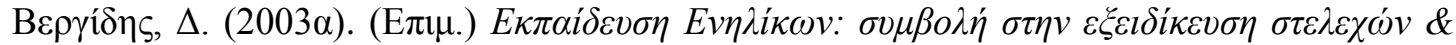

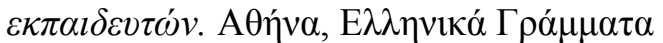

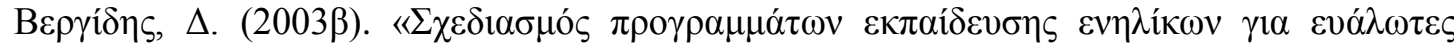

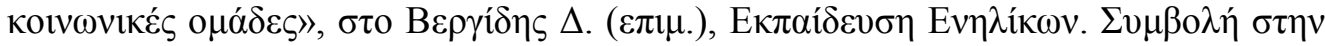

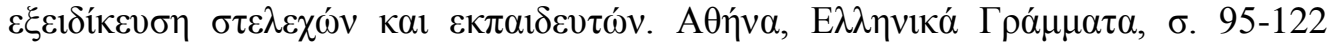

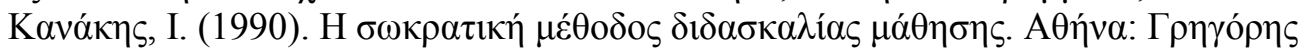

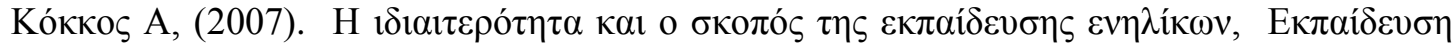

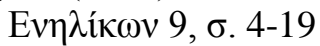

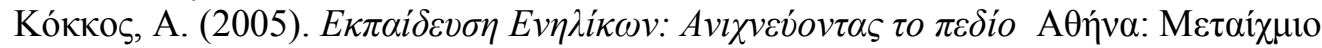

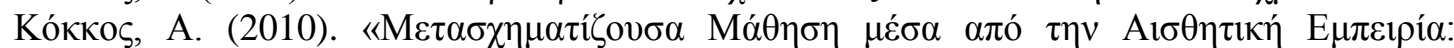

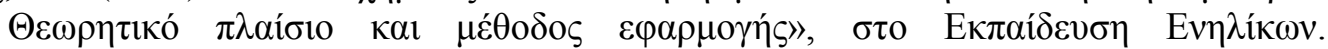

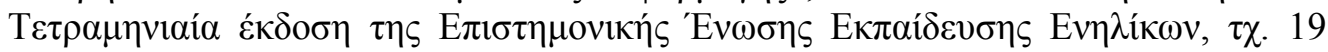

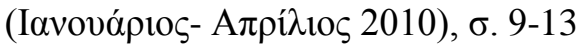




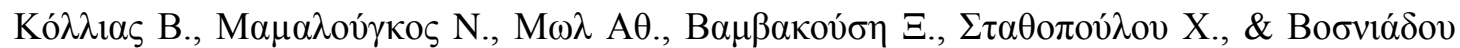

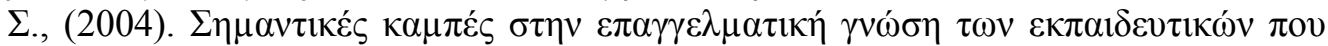

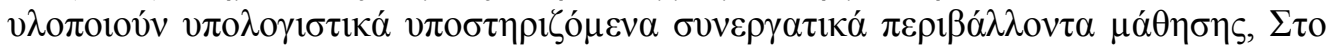

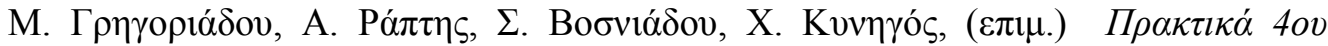

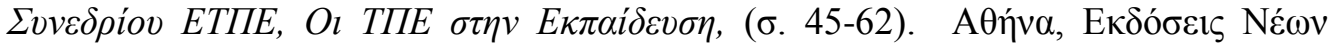

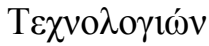

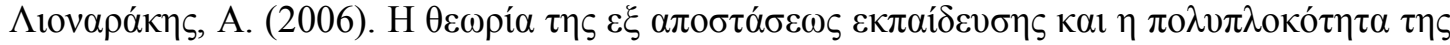

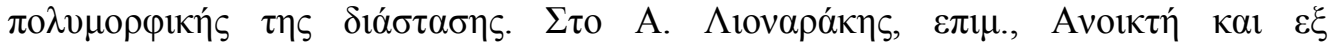

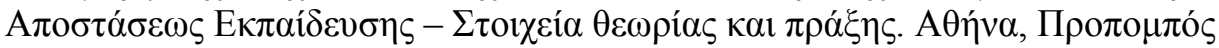

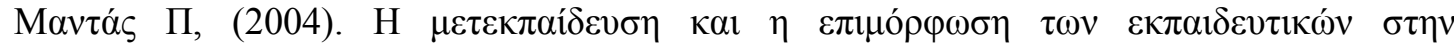
$\pi \rho \omega \tau о \beta \alpha ́ \theta \mu 1 \alpha \varepsilon \kappa \pi \alpha i ́ \delta \varepsilon v \sigma \eta$. А $\theta \dot{v} v \alpha$

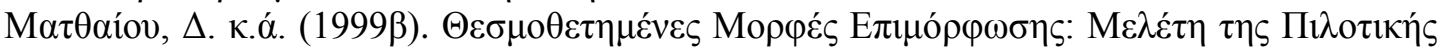

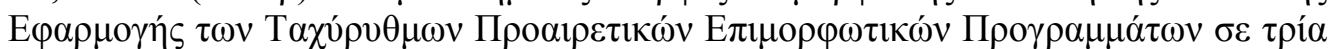

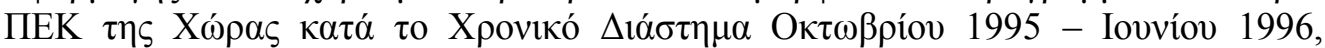

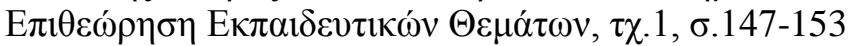

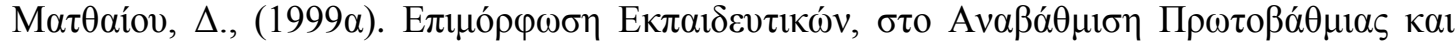

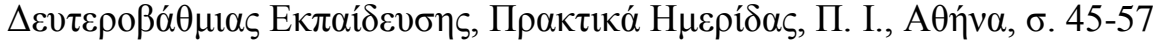

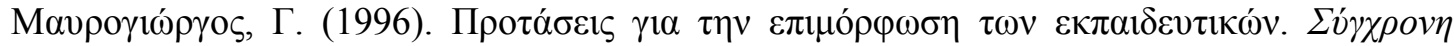

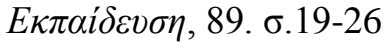

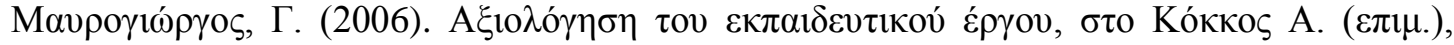

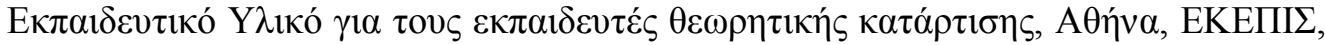

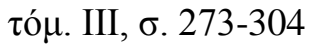

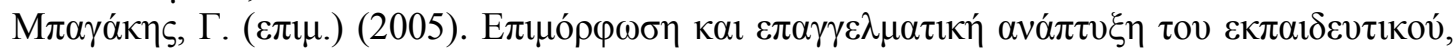

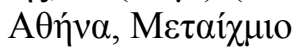

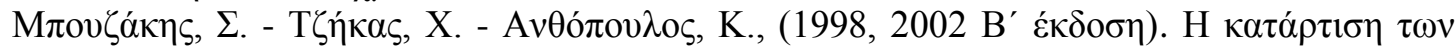

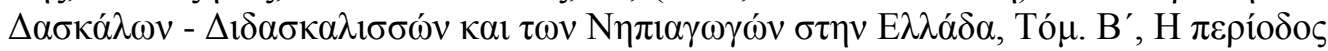

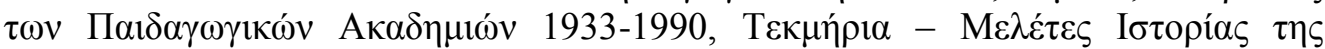

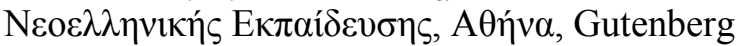

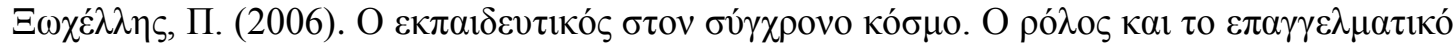

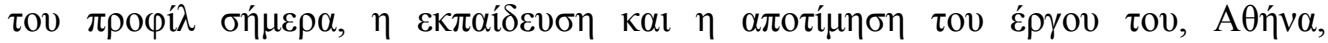

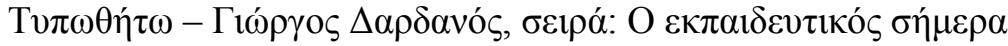

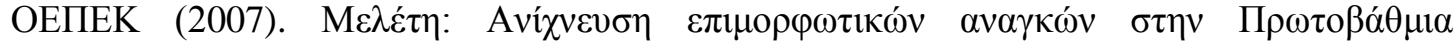

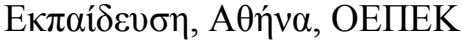

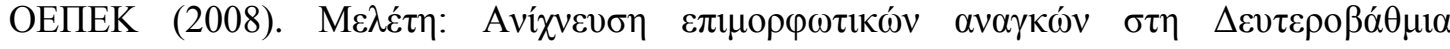

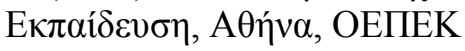

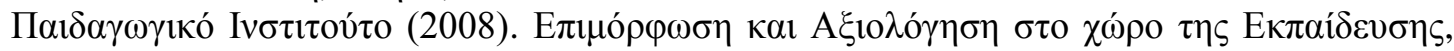

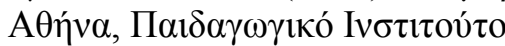

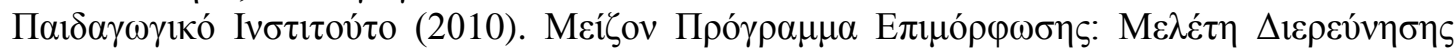

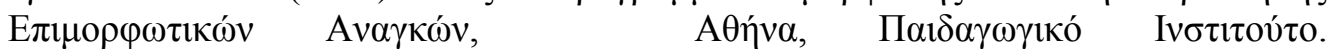
www.epimorfosi.edu.gr

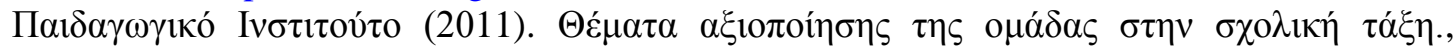

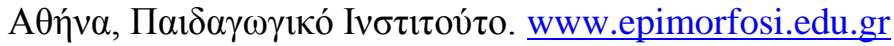

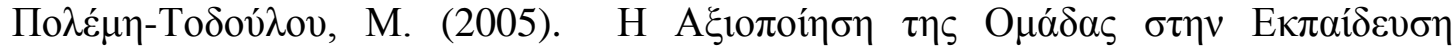

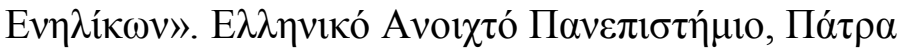

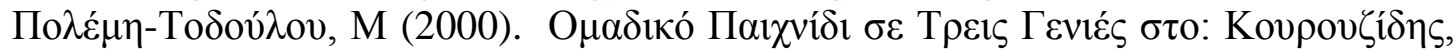

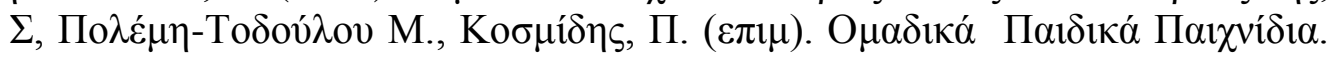

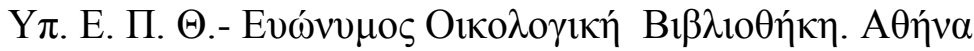

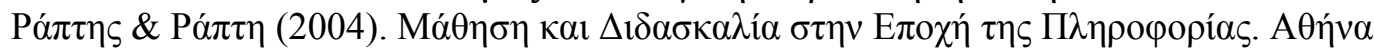

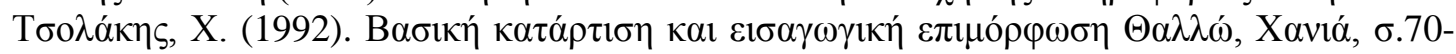
82

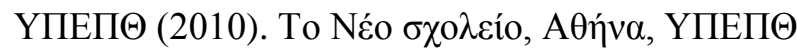

